\title{
SN 2008jb: A “LOST” CORE-COLLAPSE SUPERNOVA IN A STAR-FORMING DWARF GALAXY AT 10 Mpc*
}

\author{
J. L. Prieto ${ }^{1,2,11}$, J. C. LeE ${ }^{1,12}$, A. J. Drake ${ }^{3}$, R. McNaught $^{4}$, G. Garradd ${ }^{4}$, J. F. Beacom ${ }^{5,6,7}$, E. Beshore $^{8}$, M. Catelan $^{9}$,

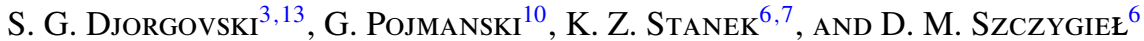 \\ ${ }^{1}$ Carnegie Observatories, 813 Santa Barbara Street, Pasadena, CA 91101, USA \\ ${ }^{2}$ Department of Astrophysical Sciences, Princeton University, Princeton, NJ 08544, USA \\ ${ }^{3}$ California Institute of Technology, 1200 E. California Blvd., CA 91225, USA \\ ${ }^{4}$ Research School of Astronomy and Astrophysics, Australian National University, Cotter Road, Weston Creek, ACT 2611, Australia \\ ${ }^{5}$ Department of Physics, The Ohio State University, 191 W. Woodruff Ave., Columbus, OH 43210, USA \\ ${ }^{6}$ Department of Astronomy, The Ohio State University, $140 \mathrm{~W}$. 18th Ave., Columbus, OH 43210, USA \\ ${ }^{7}$ Center for Cosmology and Astroparticle Physics, The Ohio State University, 191 W. Woodruff Ave., Columbus, OH 43210, USA \\ ${ }^{8}$ Department of Planetary Sciences, Lunar and Planetary Laboratory, The University of Arizona, 1629 E. University Blvd., Tucson, AZ 85721, USA \\ ${ }^{9}$ Departamento de Astronomía y Astrofísica, Pontificia Universidad Católica de Chile, Av. Vicuna Mackena 4860, 782-0436 Macul, Santiago, Chile \\ ${ }^{10}$ Warsaw University Astronomical Observatory, Al. Ujazdowskie 4, 00-478 Warsaw, Poland \\ Received 2011 July 23; accepted 2011 October 10; published 2011 December 29
}

\begin{abstract}
We present the discovery and follow-up observations of SN 2008jb, a core-collapse supernova in the southern dwarf irregular galaxy ESO $302-14\left(M_{B}=-15.3 \mathrm{mag}\right)$ at $9.6 \mathrm{Mpc}$. This nearby transient was missed by galaxy-targeted surveys and was only found in archival optical images obtained by the Catalina Real-time Transient Survey and the All-Sky Automated Survey. The well-sampled archival photometry shows that SN 2008jb was detected shortly after explosion and reached a bright optical maximum, $V_{\max } \simeq 13.6 \mathrm{mag}\left(M_{V, \max } \simeq-16.5\right)$. The shape of the light curve shows a plateau of $\sim 100$ days, followed by a drop of $\sim 1.4 \mathrm{mag}$ in the $V$ band to a slow decline with an approximate ${ }^{56} \mathrm{Co}$ decay slope. The late-time light curve is consistent with $0.04 \pm 0.01 M_{\odot}$ of ${ }^{56} \mathrm{Ni}$ synthesized in the explosion. A spectrum of the supernova obtained two years after explosion shows a broad, boxy $\mathrm{H} \alpha$ emission line, which is unusual for normal Type II-Plateau supernovae at late times. We detect the supernova in archival Spitzer and WISE images obtained 8-14 months after explosion, which show clear signs of warm (600-700 K) dust emission. The dwarf irregular host galaxy, ESO 302-14, has a low gas-phase oxygen abundance, $12+\log (\mathrm{O} / \mathrm{H})=8.2$ $\left(\sim 1 / 5 Z_{\odot}\right)$, similar to those of the Small Magellanic Cloud and the hosts of long gamma-ray bursts and luminous core-collapse supernovae. This metallicity is one of the lowest among local $(\lesssim 10 \mathrm{Mpc})$ supernova hosts. We study the host environment using GALEX far-UV, $R$-band, and $\mathrm{H} \alpha$ images and find that the supernova occurred in a large star formation complex. The morphology of the $\mathrm{H} \alpha$ emission appears as a large shell ( $R \simeq 350 \mathrm{pc}$ ) surrounding the FUV and optical emission. Using the $\mathrm{H} \alpha$-to-FUV ratio and FUV and $R$-band luminosities, we estimate an age of $\sim 9 \mathrm{Myr}$ and a total mass of $\sim 2 \times 10^{5} M_{\odot}$ for the star formation complex, assuming a single-age starburst. These properties are consistent with the expanding $\mathrm{H} \alpha$ supershells observed in many well-studied nearby dwarf galaxies, which are tell-tale signs of feedback from the cumulative effect of massive star winds and supernovae. The age estimated for the star-forming region where SN 2008jb exploded suggests a relatively high-mass progenitor star with an initial mass $M \sim 20 M_{\odot}$ and warrants further study. We discuss the implications of these findings in the study of core-collapse supernova progenitors.
\end{abstract}

Key words: galaxies: dwarf - supernovae: general - supernovae: individual (SN 2008jb)

Online-only material: color figures

\section{INTRODUCTION}

Core-collapse supernovae are energetic explosions that mark the death of stars more massive than $\approx 8-10 M_{\odot}$. They are extremely important in several areas of astrophysics, including the nucleosynthesis of chemical elements, energy feedback that affects the evolution of galaxies, the formation of compact object remnants, the production of high-energy particles, their use as tracers of recent star formation in galaxies, and their use as test cases of massive stellar evolution.

Supernovae discovered in nearby galaxies have been particularly important for testing our physical understanding of the explosions and to establish direct links between the events

\footnotetext{
* This paper includes data gathered with the $6.5 \mathrm{~m}$ Magellan telescope at Las Campanas Observatory, Chile.

${ }^{11}$ Carnegie Fellow.

12 Hubble, Carnegie-Princeton Fellow.

${ }^{13}$ Distinguished Visiting Professor, King Abdulaziz University, Jeddah 21589, Saudi Arabia.
}

and their progenitors, which puts tight constraints on stellar evolution theory. The two best-studied nearby core-collapse supernovae, the peculiar Type II SN 1987A in the LMC and the Type IIb SN 1993J in M81, had progenitors that were detected in pre-explosion images. Both were very interesting cases that challenged theoretical expectations based on stellar evolution models that predicted red supergiants as their progenitor stars: SN 1987A had a $\simeq 20 M_{\odot}$ blue supergiant progenitor star (e.g., Arnett et al. 1989) and SN 1993J had $\mathrm{a} \simeq 15 M_{\odot}$ yellow supergiant progenitor (e.g., Aldering et al. 1994), which turned out to be a massive binary system (e.g., Maund et al. 2004). After more than a decade of work, Smartt et al. (2009) presented a thorough study of nearby $(\lesssim 30 \mathrm{Mpc})$ Type II-Plateau (Type IIP) supernovae with deep pre-explosion imaging (see also Li et al. 2005), which allowed them to constrain the progenitors of Type IIP supernovae to be red supergiants with initial main-sequence masses in the range $8-17 M_{\odot}$. This result is in conflict with well-studied red supergiant samples in the Galaxy and the Magellanic 
Clouds (e.g., Levesque et al. 2006), which also contain massive supergiants with $M \simeq 20-30 M_{\odot}$ and a Salpeter initial mass function (IMF) for high-mass stars.

This "red supergiant problem" can be alleviated if high-mass red or yellow supergiants produce other spectroscopic or photometric Type II supernova subtypes (e.g., Smith et al. 2009; Elias-Rosa et al. 2009, 2010), or if high-mass supergiants experience strong winds due to pulsations (e.g., Yoon \& Cantiello 2010). Very recently, Walmswell \& Eldridge (2011) have proposed circumstellar dust as a possible solution. The problem can also be explained by failed supernovae, a hypothesis that would have several deep implications (e.g., Kochanek et al. 2008). In addition, there are known selection biases that have not been discussed in depth or accounted for. Of the 18 nearby Type II supernovae with fairly secure progenitor (either single stars, binaries, or compact clusters) detections, ${ }^{14} 2$ of the 18 events were initially discovered by the galaxy-targeted Lick Observatory Supernova Search (LOSS; Filippenko et al. 2001) and 14 of the 18 were initially discovered by dedicated amateur astronomers who mostly concentrate their search efforts on big galaxies. Only two of these supernovae were found in dwarf galaxies (SN 1987A and 2008ax) and all the Type IIPs (10 of the 18) were found in the disks of spiral galaxies, mostly in grand-design spirals.

The existing selection bias against finding nearby corecollapse supernovae in dwarf galaxies could be important because $20 \%-40 \%$ of the local star formation rate (SFR) density is in galaxies with absolute magnitude $M_{B}>-18$ mag (e.g., James et al. 2008; Young et al. 2008; Williams et al. 2011). Since metallicity is a key parameter in massive stellar evolution and stellar death (e.g., Prieto et al. 2008a; Modjaz 2011, and references therein), changes in the fractions of different Type II spectroscopic subtypes with metallicity (e.g., Arcavi et al. 2010) could affect our census of progenitors in local samples. Also, possible variations of the stellar IMF with environment (e.g., Meurer et al. 2009; but see, e.g., Myers et al. 2011, and references therein) can introduce complications to the progenitor analyses. Recently, Horiuchi et al. (2011) discussed in detail related effects of incompleteness in the existing supernova rate measurements and how they affect their use as SFR indicators.

In this paper we present the discovery, follow-up observations, and analysis of the nearby Type II SN 2008jb $\left(\alpha=03^{\mathrm{h}} 51^{\mathrm{m}} 44^{\mathrm{s}} .66\right.$ and $\left.\delta=-38^{\circ} 27^{\prime} 00^{\prime} .1\right)$ in a southern dwarf irregular galaxy at $\sim 10 \mathrm{Mpc}$ (Prieto et al. 2011). This bright 13.6 mag supernova was missed by targeted southern supernova surveys like CHASE (the Chilean Automatic Supernova sEarch; Pignata et al. 2009) and amateur searches mainly because the host galaxy ESO $302-14$ is a fairly low luminosity dwarf and was not included in the catalogs of galaxies that are surveyed for supernovae. We are able to recover the optical light curves of this supernova from two surveys that do not target individual galaxies, but rather scan large areas of the sky: the Catalina Real-time Transient Survey $^{15}$ (CRTS; Drake et al. 2009; Djorgovski et al. 2011)

\footnotetext{
14 These are SN 1987A, 1993J; SN 1961V from Kochanek et al. (2011) and Smith et al. (2011c); SN 1999ev, 2003gd, 2004A, 2004am, 2004dj, 2004et, $2005 \mathrm{cs}$, and 2008bk from the sample of Smartt et al. (2009); SN 2005gl, from Gal-Yam et al. (2007) and Gal-Yam \& Leonard (2009); SN 2008ax from Crockett et al. (2008); SN 2008cn and 2009kr from Elias-Rosa et al. (2009, 2010); SN 2009md from Fraser et al. (2011); SN 2010jl from Smith et al. (2011b); and SN 2011hd from Maund et al. (2011), Szczygiel et al. (2011), and Van Dyk et al. (2011). We do not include here low-luminosity transients with Type IIn-like spectral features and progenitor detections in the mid-infrared, like SN 2008S and NGC 300-OT (e.g., Prieto et al. 2008b).

15 http://crts.caltech.edu
}

Table 1

CRTS V-band Light Curve of SN 2008jb

\begin{tabular}{lcc}
\hline \hline $\begin{array}{l}\text { HJD-2,450,000 } \\
\text { (day) }\end{array}$ & Magnitude & $\sigma$ \\
\hline 4774.21 & $>21.0$ & \\
4794.12 & 13.58 & 0.08 \\
4804.14 & 13.86 & 0.08 \\
4835.09 & 14.26 & 0.08 \\
4864.96 & 14.61 & 0.08 \\
4896.96 & 16.03 & 0.08 \\
5074.28 & 18.36 & 0.09 \\
5114.17 & 18.81 & 0.09 \\
5147.21 & 19.53 & 0.11 \\
5163.15 & 19.06 & 0.11 \\
5180.08 & 19.81 & 0.12 \\
5208.05 & 20.23 & 0.16 \\
5238.93 & $>20.0$ & \\
\hline
\end{tabular}

and the All-Sky Automated Survey (ASAS; Pojmanski 1998). In Section 2, we discuss the observations, including the optical discovery and follow-up, archival mid-infrared (mid-IR) imaging, optical spectroscopy, and other archival data. In Section 3, we present detailed analysis and discussion of the optical and mid-IR observations. In Section 4, we discuss the results and conclusions. We adopt a distance of 9.6 Mpc to ESO 302-14 (Lee et al. 2009), estimated from the Virgo inflow corrected recession velocity and $H_{0}=75 \mathrm{~km} \mathrm{~s}^{-1} \mathrm{Mpc}^{-1}$.

\section{OBSERVATIONS}

\subsection{Optical Imaging}

SN 2008jb was discovered in CRTS archival data from the Siding Spring Survey (SSS) $0.5 \mathrm{~m}$ Schmidt telescope through the SNhunt project. ${ }^{16}$ SNhunt is an open survey for transients in nearby galaxies that uses image subtraction in images obtained by the CRTS. A new transient was recovered in 2010 October 26 (ID SNhunt12) after running the difference imaging pipeline in archival data from the SSS. SN 2008jb is first detected in unfiltered SSS images obtained on 2008 November 23, at $V \simeq 13.6$ mag. Figure 1 shows the first detection of SN 2008jb in the SSS images. It was also detected in 10 images obtained between 2008 December 3 and 2010 January 11. We used the image subtraction software described in Freedman et al. (2009) in order to obtain clean images of the supernova (see Figure 1). The image used as a template for the subtractions was a combination of images obtained in 2007. We performed point-spread function photometry using DAOPHOT (Stetson 1992) on the difference images. The final calibration of the supernova photometry was done relative to ASAS $V$-band data of stars in the field (see discussion below). Table 1 gives the $V$-band calibrated photometry from the SSS data. We also include $3 \sigma$ upper limits on the magnitudes of the supernova obtained from the images just before the first detection and after the last detection.

The supernova was also detected in archival images collected using the $7 \mathrm{~cm}$ ASAS South telescopes at Las Campanas Observatory, Chile. The first detection in ASAS South data is from 2008 November 19 (four days earlier than CRTS) at 13.9 mag in the $I$ band. The ASAS images were processed with the reduction pipeline described in Pojmanski (1998, 2002). The $V$ and $I$ magnitudes are tied to the Johnson $V$ and Cousins $I$

$\overline{16}$ http://nesssi.cacr.caltech.edu/catalina/current.html 


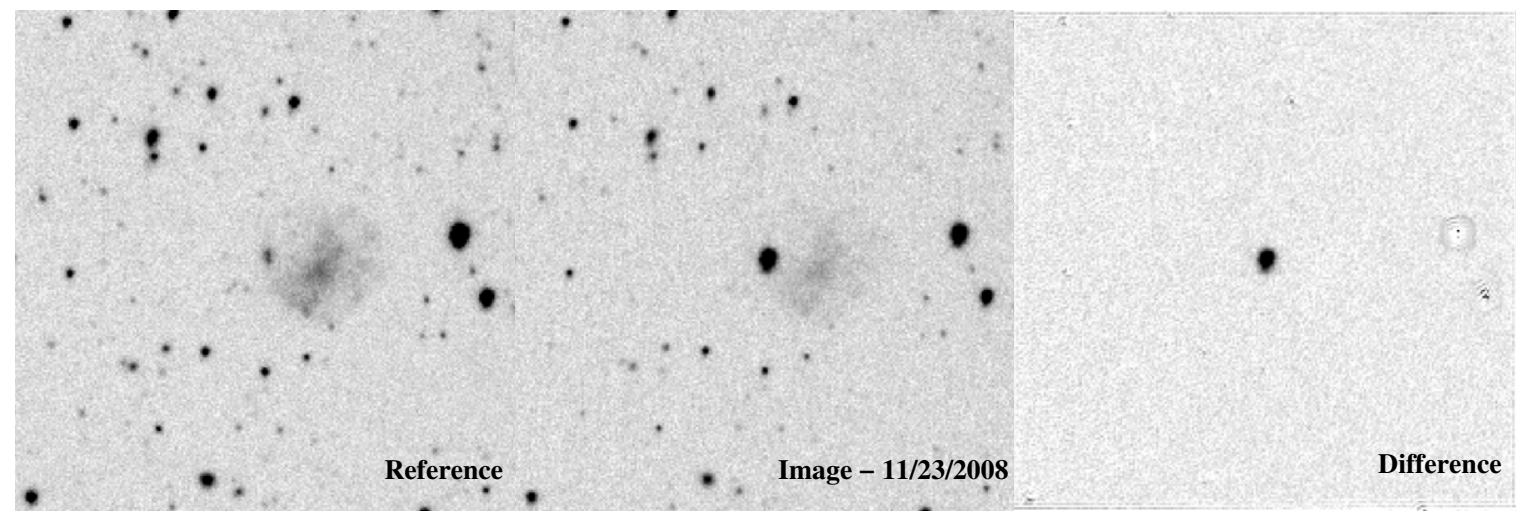

Figure 1. SSS images (3.'6 × 3.6) centered on SN 2008jb. Left panel: reference image obtained from a combination of pre-discovery images. Middle panel: first SSS post-discovery image obtained on 2008 November 23. Right panel: difference image. In all the panels, north is up and east is to the left.

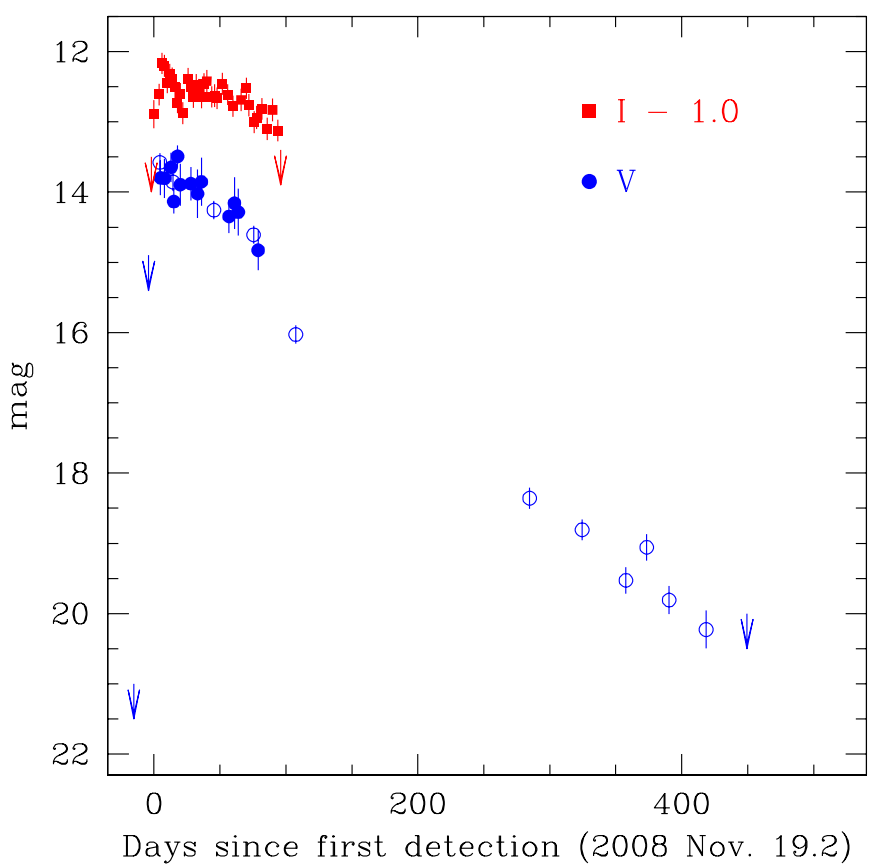

Figure 2. Light curves of SN 2008jb obtained from CRTS (open symbols; unfiltered, calibrated in the $V$ band) and ASAS (filled symbols; $V$ and $I$ bands) photometric data. The arrows are $3 \sigma$ upper limits on the magnitudes.

(A color version of this figure is available in the online journal.)

scale using Tycho (Høg et al. 2000) and Landolt (Landolt 1983) standard stars. We used aperture photometry (2 pixel radius) from the normal reduction pipeline to obtain the magnitudes of the supernova in ASAS images and estimated $3 \sigma$ upper limits before and after the first and last detection epochs, respectively. Unlike the case of the SSS images where there is contamination from the nearby star-forming region at faint magnitudes, the much shallower ASAS data $(V \lesssim 15 \mathrm{mag}$ ) do not require image subtraction. The ASAS $V$ and $I$ magnitudes of SN 2008jb are presented in Table 2. Figure 2 shows the light curve of the supernova from ASAS and CRTS data.

We obtained late-time observations of SN 2008jb on 2010 November 7 using IMACS (Dressler et al. 2006) on the $6.5 \mathrm{~m}$ Magellan I (Baade) telescope at Las Campanas Observatory. We obtained $3 \times 200 \mathrm{~s}$ images of the field of SN 2008jb with the $f / 2$ camera $\left(0\right.$.'2 pixel $\left.^{-1}\right)$ using the $R$-band filter under good weather and seeing conditions $\left(00^{\prime} 7-0.8\right)$. Since we lack the deep template image necessary to obtain the supernova flux at late times, these data are only used to study the host galaxy environment. We plan to obtain more late-time observations and the analysis of these data will be presented in a future publication.

\subsection{Optical Spectroscopy}

We gathered spectroscopic observations of SN 2008jb on 2011 January 6 using IMACS on the $6.5 \mathrm{~m}$ Baade telescope at Las Campanas Observatory. The observations consisted of $3 \times 1800 \mathrm{~s}$ spectra obtained using the $3001 \mathrm{~mm}^{-1}$ grism (range 4000-9000 $\AA$ ) and a 0.'9 slit aligned at the parallactic angle, obtained under clear weather and good seeing (0'.7). This setup gives an FWHM resolution of $4 \AA$ at $6500 \AA$. The data were reduced using standard techniques in IRAF, which included basic data reduction (overscan and bias subtraction, flat-fielding), one-dimensional spectrum extraction, wavelength calibration using a HeNeAr arclamp obtained after the science observations, and flux calibration using a flux standard observed on the same night. The spectrum goes through two CCD detectors separated by a gap of $\sim 74 \AA$. We used a simple linear interpolation at the edges of the CCDs. The final spectrum of SN 2008jb is shown in Figure 3.

We also obtained the spectrum of an $\mathrm{H}$ II region in the host galaxy at $\sim 150 \mathrm{pc}\left(\sim 3^{\prime \prime}\right)$ south of the supernova position on 2011 January 2 with the Wide-Field Reimaging CCD Camera (WFCCD) on the $2.5 \mathrm{~m}$ du Pont telescope at Las Campanas Observatory. The spectrum consisted of $3 \times 1800 \mathrm{~s}$ exposures with the $4001 \mathrm{~mm}^{-1}$ grism, which gives an FWHM resolution of $8 \AA$ and continuous coverage in the 3800-9200 $\AA$ range. The data were reduced using standard tasks in IRAF similar to the reduction procedure of the IMACS supernova spectrum. The final spectrum of the $\mathrm{HII}$ region is shown in Figure 4. We measured the fluxes of the most prominent emission lines in the spectrum using Gaussian profiles and a custom Perl Data Language fitting routine, including the Balmer recombination lines $(\mathrm{H} \beta \lambda 4862$ and $\mathrm{H} \alpha \lambda$ 6563) and forbidden emission lines ([O III] $\lambda \lambda$ 4959, 5007, [S II] $\lambda \lambda$ 6713, 6731, and [N II] $\lambda$ 6583). The fluxes of the optical emission lines are presented in Table 3.

\subsection{Mid-infrared Imaging}

The host galaxy of SN 2008jb, ESO 302-14, was observed with Spitzer/IRAC (Fazio et al. 2004) using the 3.6 and $4.5 \mu \mathrm{m}$ channels in warm Spitzer observations obtained as part of the Spitzer Survey of Stellar Structure in Galaxies (Sheth et al. 2010). Two epochs of imaging were obtained during Cycle 6 (PID 61060) separated by $\sim 1$ month, in 2009 September 2 
Table 2

ASAS Light Curve of SN 2008jb

\begin{tabular}{|c|c|c|}
\hline $\begin{array}{l}\text { HJD-2,450,000 } \\
\text { (day) }\end{array}$ & Magnitude & $\sigma$ \\
\hline & $V$ & \\
\hline 4785.66 & $>14.9$ & \\
\hline 4794.68 & 13.80 & 0.25 \\
\hline 4797.69 & 13.80 & 0.28 \\
\hline 4802.64 & 13.65 & 0.21 \\
\hline 4804.73 & 14.13 & 0.17 \\
\hline 4807.59 & 13.49 & 0.16 \\
\hline 4809.65 & 13.89 & 0.30 \\
\hline 4817.64 & 13.88 & 0.24 \\
\hline 4822.77 & 14.02 & 0.35 \\
\hline 4825.65 & 13.85 & 0.34 \\
\hline 4846.61 & 14.35 & 0.24 \\
\hline 4850.73 & 14.16 & 0.37 \\
\hline 4853.64 & 14.29 & 0.33 \\
\hline \multirow[t]{2}{*}{4868.60} & 14.83 & 0.28 \\
\hline & $I$ & \\
\hline 4787.65 & $>14.5$ & \\
\hline 4789.70 & 13.89 & 0.20 \\
\hline 4793.70 & 13.61 & 0.15 \\
\hline 4795.70 & 13.17 & 0.15 \\
\hline 4797.71 & 13.20 & 0.16 \\
\hline 4799.72 & 13.45 & 0.15 \\
\hline 4801.68 & 13.32 & 0.15 \\
\hline 4803.65 & 13.38 & 0.16 \\
\hline 4805.65 & 13.50 & 0.16 \\
\hline 4807.64 & 13.72 & 0.15 \\
\hline 4809.65 & 13.60 & 0.15 \\
\hline 4811.68 & 13.88 & 0.16 \\
\hline 4815.67 & 13.39 & 0.16 \\
\hline 4817.67 & 13.51 & 0.15 \\
\hline 4819.67 & 13.65 & 0.15 \\
\hline 4821.66 & 13.47 & 0.15 \\
\hline 4823.66 & 13.54 & 0.15 \\
\hline 4825.66 & 13.65 & 0.16 \\
\hline 4827.67 & 13.46 & 0.15 \\
\hline 4829.68 & 13.42 & 0.16 \\
\hline 4833.64 & 13.64 & 0.15 \\
\hline 4835.62 & 13.63 & 0.16 \\
\hline 4837.62 & 13.66 & 0.15 \\
\hline 4841.64 & 13.46 & 0.15 \\
\hline 4845.64 & 13.61 & 0.15 \\
\hline 4849.63 & 13.78 & 0.15 \\
\hline 4855.63 & 13.69 & 0.16 \\
\hline 4859.62 & 13.52 & 0.15 \\
\hline 4861.62 & 13.76 & 0.16 \\
\hline 4865.61 & 14.00 & 0.15 \\
\hline 4867.60 & 13.94 & 0.17 \\
\hline 4871.61 & 13.81 & 0.15 \\
\hline 4875.61 & 14.10 & 0.16 \\
\hline 4879.60 & 13.83 & 0.16 \\
\hline 4883.59 & 14.12 & 0.15 \\
\hline 4885.60 & $>14.4$ & \\
\hline
\end{tabular}

and October 10. We retrieved the post-BCD images of ESO 302-14 from the Spitzer Heritage Archive, which are fully reduced and flux-calibrated. The supernova is clearly detected as a bright, variable mid-IR source in these images, at a position consistent with the optical coordinates. The mid-IR detections are shown in Figure 5. We measured the 3.6 and $4.5 \mu \mathrm{m}$ fluxes of the source using aperture photometry with a 2".4 aperture radius $(2.4-7$ ". 2 annulus for sky determination) and applied

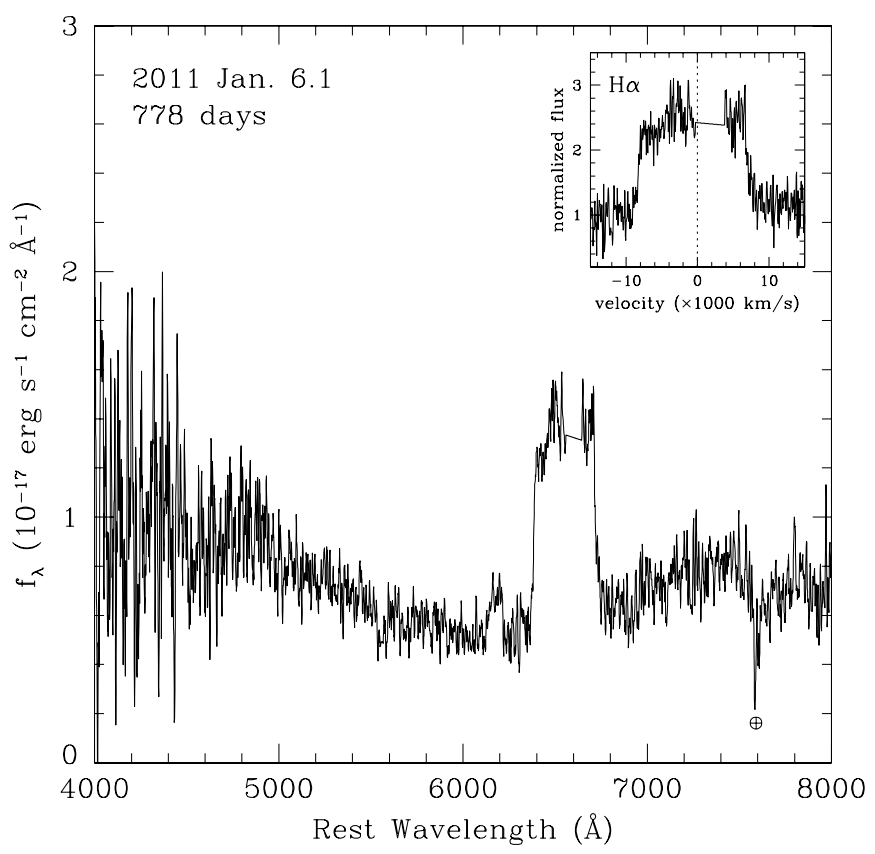

Figure 3. Late-time optical spectrum of SN 2008jb obtained with Magellan/IMACS on 2011 January 6.1, 778 days after the first detection of the supernova with ASAS. The wavelength has been corrected to the rest frame using the recession velocity of the host galaxy $\left(v=872 \mathrm{~km} \mathrm{~s}^{-1}\right)$. The spectrum is dominated by a broad, flat-topped $\mathrm{H} \alpha$ emission line $\left(\mathrm{FWHM} \simeq 14000 \mathrm{~km} \mathrm{~s}^{-1}\right.$ ) shown in the inset panel. We have linearly interpolated the fluxes in the chip gap between 6588 and $6662 \AA$ (observed wavelength). The fluxes have been corrected for Galactic extinction and smoothed using a five-pixel boxcar.

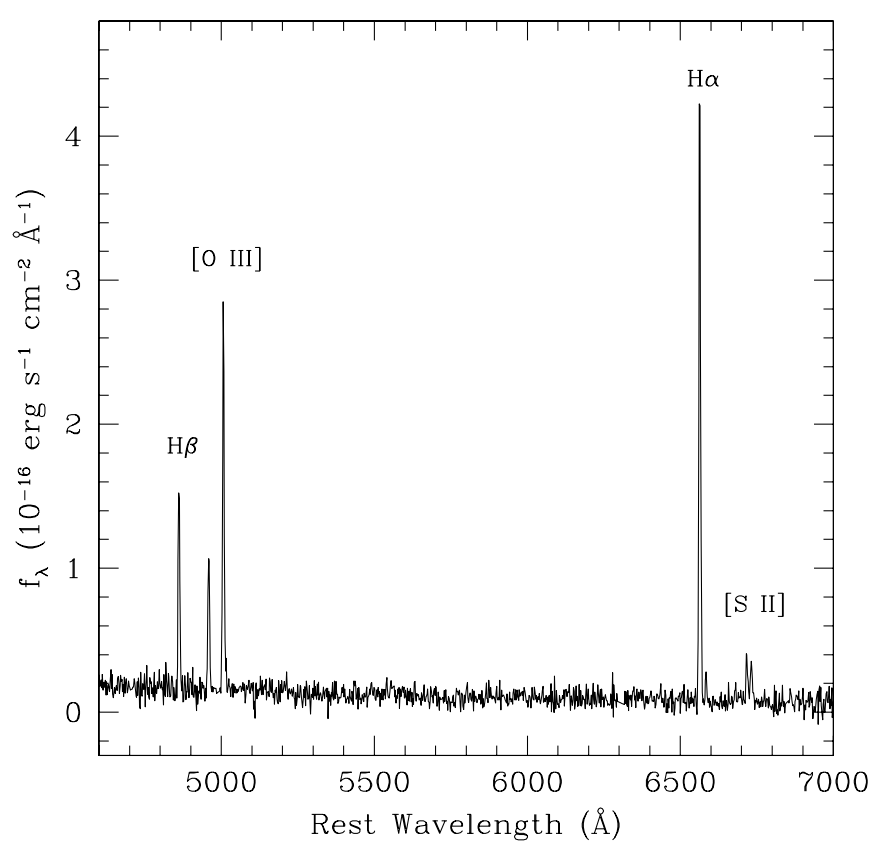

Figure 4. Optical spectrum obtained with du Pont/WFCCD of a nearby H II region in the dwarf irregular host galaxy of SN 2008jb, ESO 302-14. The spectrum shows Balmer recombination lines $(\mathrm{H} \alpha$ and $\mathrm{H} \beta)$ and forbidden emission lines ([O III] $\lambda \lambda$ 4959, 5007, [S II] $\lambda \lambda$ 6713, 6731, and weak [N II] $\lambda$ 6583). The wavelength has been corrected to the rest frame using the recession velocity of the host galaxy. The fluxes have been corrected for Galactic extinction.

the aperture corrections and flux conversion factors listed in the IRAC Instrument Handbook. The IRAC fluxes are presented in Table 4. 


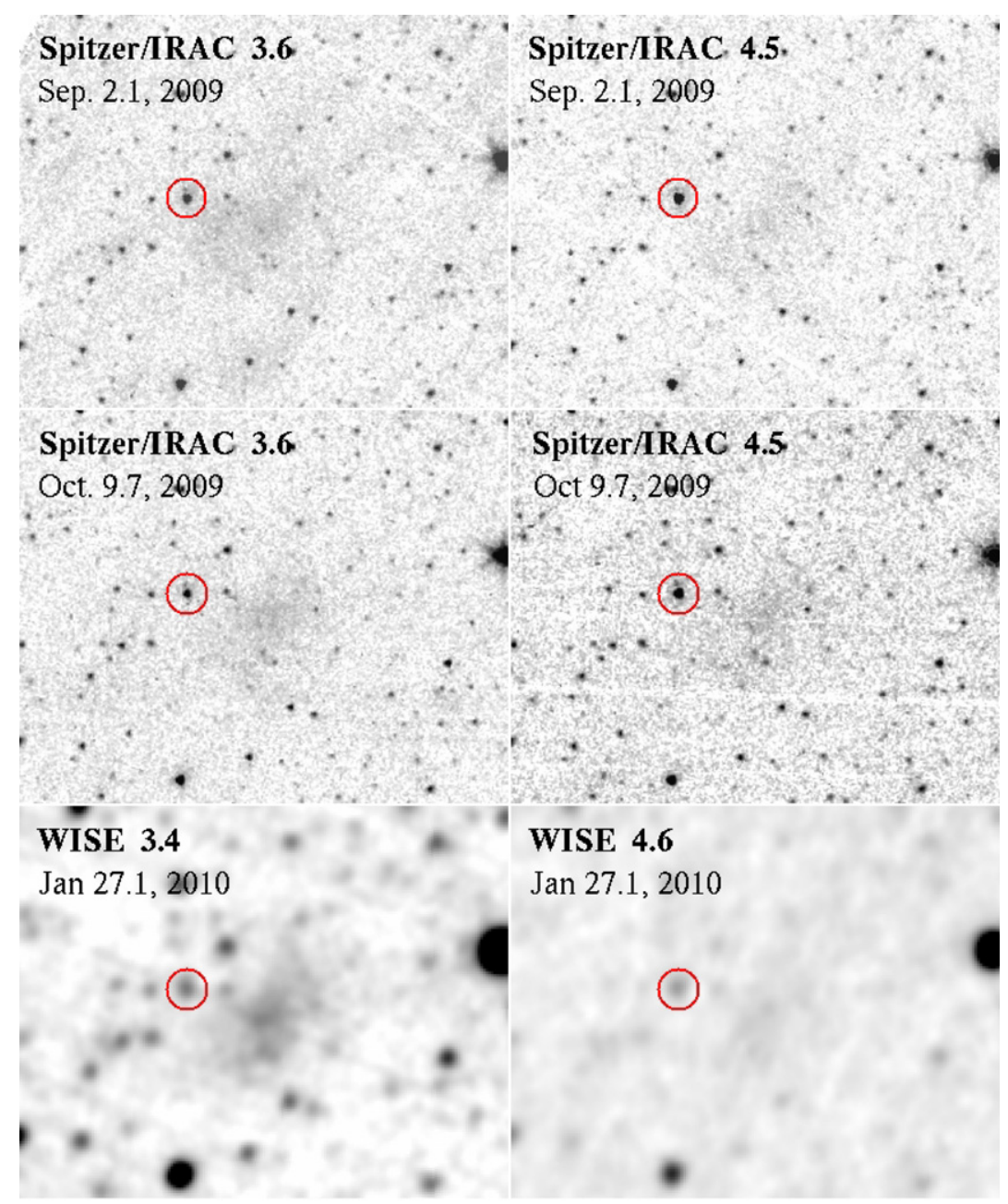

Figure 5. Spitzer/IRAC (3.6 $\mu \mathrm{m}$ and $4.5 \mu \mathrm{m})$ and WISE $(3.4 \mu \mathrm{m}$ and $4.6 \mu \mathrm{m})$ images showing the late-time detections of SN 2008jb (red circle) at three different epochs. Each 4.1 $\times$ 3.3 panel is centered on the host galaxy of SN 2008jb. In all panels, north is up and east is to the left.

(A color version of this figure is available in the online journal.)

Table 3

Emission Line Fluxes of H II Region in ESO 302-14

\begin{tabular}{lc}
\hline \hline Line & $\begin{array}{c}\text { Flux }^{\mathrm{a}} \\
\left(10^{-15} \mathrm{erg} \mathrm{s}^{-1} \mathrm{~cm}^{-2}\right)\end{array}$ \\
\hline $\mathrm{H} \beta$ & $0.94 \pm 0.09$ \\
{$[\mathrm{O}$ III] 4959} & $0.67 \pm 0.07$ \\
{$[\mathrm{O}$ III 5007} & $1.68 \pm 0.15$ \\
$\mathrm{H} \alpha$ & $2.84 \pm 0.21$ \\
{$[\mathrm{~N} \mathrm{II}] 6583$} & $0.12 \pm 0.02$ \\
{$\left[\mathrm{~S} \mathrm{II}_{\text {II }} 6713\right.$} & $0.22 \pm 0.03$ \\
{$\left[\mathrm{~S} \mathrm{II}_{6}\right] 6731$} & $0.22 \pm 0.03$ \\
\hline
\end{tabular}

Notes. ${ }^{\text {a }}$ Fluxes have been corrected by Galactic $E(B-V)_{\mathrm{MW}}=$ 0.009 mag using CCM reddening law.

The WISE (Wright et al. 2010) preliminary data release includes observations of the field of SN 2008jb obtained at 3.4, 4.6, 12, and $22 \mu \mathrm{m}$ between 2010 January 23 and January 30. We searched for the WISE data in the NASA/IPAC Infrared Science Archive. The supernova is clearly detected at 3.4 and $4.6 \mu \mathrm{m}$ (see Figure 5), marginally detected at $12 \mu \mathrm{m}$, and undetected at $22 \mu \mathrm{m}$. We retrieved the photometry of source J035144.63-382700.6 from the WISE catalog and list the fluxes in Table 4 . We did not attempt to measure photometry in
Table 4

Spitzer and WISE Photometry of SN 2008jb

\begin{tabular}{lcccc}
\hline \hline HJD & Band & $\begin{array}{c}\text { Flux } \\
(\mathrm{mJy})\end{array}$ & $\begin{array}{c}\text { Vega } \\
(\mathrm{mag})\end{array}$ & Instrument \\
\hline 2455076.12 & $3.6 \mu \mathrm{m}$ & $0.47 \pm 0.01$ & $14.43 \pm 0.03$ & Spitzer $/$ IRAC \\
2455076.12 & $4.5 \mu \mathrm{m}$ & $0.88 \pm 0.03$ & $13.28 \pm 0.03$ & Spitzer $/$ IRAC \\
2455113.71 & $3.6 \mu \mathrm{m}$ & $0.32 \pm 0.01$ & $14.87 \pm 0.03$ & Spitzer $/$ IRAC \\
2455113.71 & $4.5 \mu \mathrm{m}$ & $0.59 \pm 0.02$ & $13.71 \pm 0.03$ & Spitzer $/$ IRAC \\
2455223.63 & $3.4 \mu \mathrm{m}$ & $0.10 \pm 0.01$ & $16.20 \pm 0.07$ & WISE \\
2455223.63 & $4.6 \mu \mathrm{m}$ & $0.19 \pm 0.01$ & $14.88 \pm 0.07$ & WISE \\
2455223.63 & $12 \mu \mathrm{m}$ & $0.25 \pm 0.09$ & $12.75 \pm 0.40$ & WISE \\
2455223.63 & $22 \mu \mathrm{m}$ & $<1.8$ & $>9.18$ & WISE \\
\hline
\end{tabular}

Notes. Spitzer data are from warm Spitzer/IRAC program 61060 (PI: K. Sheth).

individual WISE images, but rely on the catalog fluxes measured from the combined data. The effective date of the observations is 2010 January 27.

\subsection{Other Archival Data}

We searched for other existing archival data of ESO 302-14 obtained before the discovery of SN 2008jb to help characterize the progenitor and galaxy environment. The Survey for Ionization in Neutral Gas Galaxies (SINGG; Meurer et al. 2006) 
Table 5

Properties of SN 2008jb

\begin{tabular}{|c|c|c|}
\hline Parameter & Value & Note/Reference \\
\hline SN name & SN 2008jb & CBET 2771 \\
\hline R.A. (J2000) & $03^{\mathrm{h}} 51^{\mathrm{m}} 44^{\mathrm{s}} .66$ & \\
\hline Decl. (J2000) & $-38^{\circ} 27^{\prime} 00^{\prime \prime} 1$ & \\
\hline Spectroscopic Type & II & Broad $\mathrm{H} \alpha$ in spectrum \\
\hline$E(B-V)_{\mathrm{MW}}$ & $0.009 \mathrm{mag}$ & Schlegel et al.(1998) \\
\hline$E(B-V)_{\text {host }}$ & $0.06 \pm 0.02 \mathrm{mag}$ & From Balmer decrement \\
\hline $\mathrm{HJD}_{\exp }$ & 2454782.0 & Explosion time \\
\hline$V_{\max } / I_{\max }$ & $13.59 / 13.38 \mathrm{mag}$ & $V$ and $I$ at maximum \\
\hline$M_{V, \max }$ & $-16.52 \mathrm{mag}$ & Absolute $V$ mag at maximum \\
\hline$(V-I)_{0, \max }$ & $0.13 \mathrm{mag}$ & Unreddened color at maximum \\
\hline$V_{\text {mid }} / I_{\text {mid }}$ & $14.23 / 13.65 \mathrm{mag}$ & $V$ and $I$ at mid plateau \\
\hline$M_{V, \text { mid }}$ & $-15.87 \mathrm{mag}$ & Absolute $V$ mag at mid plateau \\
\hline$(V-I)_{0, \text { mid }}$ & $0.50 \mathrm{mag}$ & Unreddened color at mid plateau \\
\hline Duration of "plateau" & $\sim 95$ days & From $I$ \\
\hline Linear decline slope ( $<100$ days) & $0.013 / 0.007 \mathrm{mag} \mathrm{day}^{-1}$ & $V$ and $I$ \\
\hline Linear decline slope (>100 days) & $0.013 \mathrm{mag} \mathrm{day}^{-1}$ & $V$ \\
\hline
\end{tabular}

Table 6

SN 2008jb Host Galaxy Properties

\begin{tabular}{lcl}
\hline \hline Parameter & Value & \multicolumn{1}{c}{ Note/Reference } \\
\hline Name & ESO 302-14 & \\
R.A. (J2000) & $03^{\mathrm{h}} 51^{\mathrm{m}} 40^{\mathrm{s}} .8$ & Paturel et al. (2003) \\
Decl. (J2000) & $-38^{\circ} 27^{\prime} 12^{\prime \prime} .4$ & Paturel et al. (2003) \\
Morphological Type & $\mathrm{IB}(\mathrm{s}) \mathrm{m}$ & RC3 \\
Heliocentric velocity & $872 \mathrm{~km} \mathrm{~s}^{-1}$ & Koribalski et al. (2004) \\
Distance modulus & $29.91 \mathrm{mag}$ & Using $d_{\text {flow }}=9.6 \mathrm{Mpc}$ \\
$B_{\text {total }}$ & $14.84 \pm 0.09 \mathrm{mag}$ & Lauberts \& Valentijn (1989) \\
$R_{\text {total }}$ & $14.54 \pm 0.09 \mathrm{mag}$ & Lauberts \& Valentijn (1989) \\
$M_{B}$ & $-15.33 \mathrm{mag}$ & Absolute $B$ mag \\
(B $-R)_{0}$ & $0.20 \mathrm{mag}$ & Unreddened color \\
Oxygen abundance 1 & $8.21 \pm 0.03$ & PP04 O3N2 method \\
Oxygen abundance 2 & $8.13 \pm 0.03$ & PP04 N2 method \\
Star formation rate & $0.03 M_{\odot} \mathrm{yr}^{-1}$ & Lee et al.(2009) \\
Stellar mass & $4.1 \times 10^{7} M_{\odot}$ & $M / L$ from Bell \& de Jong (2001) \\
H I mass & $2.5 \times 10^{8} M_{\odot}$ & Meurer et al.(2006) \\
\hline
\end{tabular}

obtained $R$-band and $\mathrm{H} \alpha$ narrowband images of the host galaxy using the CTIO $1.5 \mathrm{~m}$ telescope on 2000 October 28 (seeing was $1^{\prime \prime}$ in $R$ ). We retrieved calibrated images from SINGG (including $\mathrm{H} \alpha$-subtracted images) through the NASA Extragalactic Database.

The GALEX (Martin et al. 2005) ultraviolet space telescope observed the field of ESO 302-14 on 2004 November 18. We retrieved calibrated NUV and FUV images (GR6 data release) from the GALEX online archive.

We also searched for pre-explosion imaging data in the Hubble Space Telescope (HST), Gemini, and ESO archives. Unfortunately, there are no deep pre-explosion images obtained with these facilities.

\section{ANALYSIS}

In the following sections, we present analysis and discussion of the results from the light curves, spectra, mid-IR emission, and host galaxy environment. We present a summary of derived physical properties obtained from the optical light curves and host galaxy in Tables 5 and 6, respectively.

\subsection{Light Curve}

In order to make light curve comparisons and derive intrinsic physical properties, we first need to estimate the total reddening along the line of sight to the supernova. For the Galactic reddening, we use a CCM reddening law with $R_{V}=3.1$ (Cardelli et al. 1989) and $E(B-V)_{\mathrm{MW}}=0.009$ mag from the reddening maps of Schlegel et al. (1998). We estimate the intrinsic reddening in the host galaxy using the Balmer decrement measured from the spectrum of the $\mathrm{H}$ II region in ESO 302-14 (see Table 3). We assume an intrinsic case B recombination Balmer flux ratio $\mathrm{H} \alpha / \mathrm{H} \beta=2.86$, which is appropriate for an $\mathrm{H}$ II region at a typical electron temperature and density (Storey \& Hummer 1995). Then, we assume an SMC reddening law from Gordon et al. (2003) for the host galaxy, which is appropriate given its SFR, absolute magnitude, and metallicity (see Section 3.4). The resulting intrinsic color excess is $E(B-V)_{\text {host }}=0.06 \pm 0.02 \mathrm{mag}$ and extinction $A_{V}=0.16 \pm 0.06 \mathrm{mag}$, which we will use in all subsequent analysis. The values obtained using an LMC reddening law, $E(B-$ $V)_{\text {host }}=0.05 \mathrm{mag}$ and $A_{V}=0.17 \mathrm{mag}$, and Galactic CCM reddening law, $E(B-V)_{\text {host }}=0.06 \mathrm{mag}$ and $A_{V}=0.19 \mathrm{mag}$, are consistent with our adopted reddening. We note that for $\mathrm{H}$ II regions its usually assumed $E(B-V)_{\star}=0.44 E(B-V)_{\text {gas }}$ (Calzetti 2001), but we adopt conservatively $E(B-V)_{\text {host }} \simeq$ $E(B-V)_{\text {gas }}$ because of the young age of the region (see Section 3.4).

The $V$ - and $I$-band light curves of SN 2008jb resemble those of Type IIP supernovae, the most common kind of corecollapse events in nearby galaxies (e.g., Li et al. 2011). In Figure 6, we compare the absolute $V$-band light curves of different core-collapse supernovae with SN 2008jb, including the low-luminosity Type IIP 2005cs (Pastorello et al. 2009a), the luminous Type IIP 2004et (Maguire et al. 2010), and the low-luminosity Type IIL 1999ga (Pastorello et al. 2009b). The absolute magnitudes of SN 2008jb lie in between SN 2005cs and SN 2004et, and appear consistent with SN 1999ga and normal Type IIP supernovae (e.g., Li et al. 2011). In the comparison, we have adopted the host distances, total extinctions, and explosion times presented in the published studies. We estimate an approximate explosion time for SN 2008jb as the midpoint between the last pre-discovery $V$-band non-detection from CRTS and the first $I$-band detection from ASAS, which gives $\mathrm{HJD}_{\mathrm{exp}} \simeq 2454782.0$ (2008 November 11).

The ASAS data sample well the initial plateau, which lasts $\sim 95$ days in the $I$ band. This plateau duration is in the observed range of plateaus in Type IIP supernovae, typically between 80-120 days (e.g., Bersten \& Hamuy 2009). The I-band light 


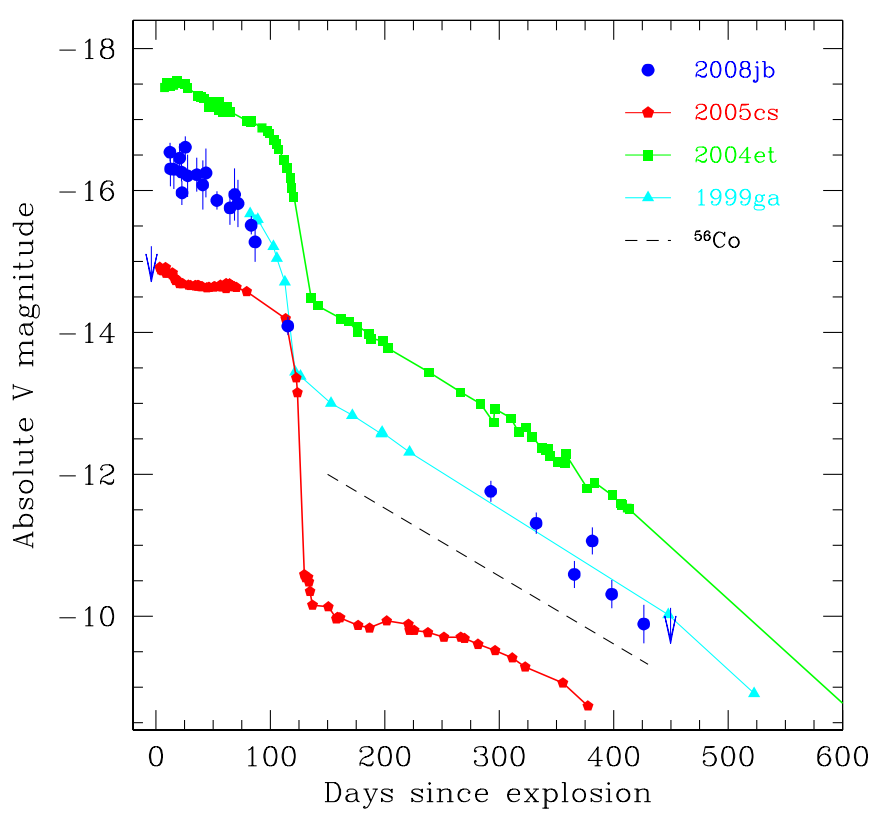

Figure 6. Absolute magnitude light curves in the $V$ band of Type II supernovae compared with SN 2008jb (filled circles). The comparison light curves are: the low-luminosity Type IIP 2005cs (filled pentagons; Pastorello et al. 2009a), the luminous Type IIP 2004et (filled squares; Maguire et al. 2010), and the low-luminosity Type IIL 1999ga (filled triangles; Pastorello et al. 2009b). The dashed line shows the slope of the ${ }^{56}$ Co decay $\left(0.01\right.$ mag day $\left.^{-1}\right)$. The magnitudes have been corrected by Galactic and internal extinction in each case.

(A color version of this figure is available in the online journal.)

curve shows a slow linear decay of $0.007 \mathrm{mag} \mathrm{day}^{-1}$ and the $V$-band light curve declines faster at $0.013 \mathrm{mag} \mathrm{day}^{-1}$ in the initial phase, which is slightly faster than well-studied Type IIP supernovae where the initial decay slope in the plateau is slower in redder bands (e.g., Poznanski et al. 2009; D’Andrea et al. 2010). It is interesting to note the similarities of the initial light curve decline of SN 2008jb with the light curve of the low-luminosity Type IIL SN 1999ga (Pastorello et al. 2009b), as shown in Figure 6. The $V$-band light curves of Type IIL supernovae typically have faster initial decline slopes than SN 2008jb and SN 1999ga (e.g., Barbon et al. 1979; Figure 3 of Pastorello et al. 2009b).

The absolute magnitudes close to the explosion date are $M_{V, \max } \simeq-16.52 \mathrm{mag}$ and $M_{I, \max } \simeq-16.65 \mathrm{mag}$. At midplateau ( $~ 50$ days), the absolute magnitudes are $M_{V \text {, mid }} \simeq$ $-15.87 \mathrm{mag}$ and $M_{I, \mathrm{mid}} \simeq-16.37 \mathrm{mag}$. The evolution of the $V-I$ color, from $(V-I)_{0} \simeq 0.13$ mag close to explosion to $(V-I)_{0} \simeq 0.50$ mag at mid-plateau, is also fairly consistent with the evolution of well-studied Type IIP supernovae, which traces small changes in the color temperature of the photosphere (e.g., Hamuy et al. 2001). It is worth noting that the unreddened color at mid-plateau of SN $2008 \mathrm{jb}$ is very close to the $(V-I)_{0}=$ 0.53 mag ridgeline that Nugent et al. (2006) derived from a large sample of Type IIP supernovae, supporting our reddening estimation.

After the $\sim 100$ day plateau, the light curve drops $\sim 1.4$ mag in 32 days (at $\sim 0.044 \mathrm{mag} \mathrm{day}^{-1}$ ) to a late-time linear decay of $0.013 \mathrm{mag} \mathrm{day}^{-1}$. We do not have $I$-band observations that show the transition phase, but the ASAS non-detections at these epochs are consistent with the $V$-band light curve shape. The $V$-band drop to a late-time linear decay is seen in all Type IIP supernovae, but it is typically stronger ( $\gtrsim 2 \mathrm{mag}$ in the $V$ band; e.g., Maguire et al. 2010). In Figure 6, we show that the late-time linear decay of SN 2008jb is similar to other Type II supernovae and reasonably consistent with the ${ }^{56} \mathrm{Co}$ to ${ }^{56} \mathrm{Fe}$ radioactive decay slope.

Hamuy (2003) studied the physical properties of a sample of well-observed Type II supernovae and derived the ${ }^{56} \mathrm{Ni}$ masses produced in the explosions from their late-time light curves, assuming full trapping of $\gamma$-ray photons produced by the radioactive decay of ${ }^{56} \mathrm{Co}$. Using Equation (1) in his study evaluated at day 115 after explosion (the first light curve point at the radioactive tail), we obtain an ${ }^{56} \mathrm{Ni}$ mass of $0.04 \pm 0.01 \mathrm{M}_{\odot}$. This result assumes a bolometric correction of $0.26 \pm 0.06 \mathrm{mag}$ in the $V$ band, which is calculated from the well-studied SN 1987A and SN 1999em at nebular phases (Hamuy 2003). The estimated uncertainty does not include a systematic error in the distance to the host.

\subsection{Spectrum}

The late-time spectrum of $\mathrm{SN} 2008 \mathrm{jb}$, obtained $\sim 2$ years after the explosion, shows a prominent $\mathrm{H} \alpha$ emission feature (see Figure 3), supporting the classification of this transient as a Type II. The H $\alpha$ feature is broad $(\mathrm{EW} \sim 480 \AA)$, flattopped, boxy, and blueshifted (centered at $\sim-1300 \mathrm{~km} \mathrm{~s}^{-1}$ ), with the blue edge of the line at $-8700 \mathrm{~km} \mathrm{~s}^{-1}$ and red edge at $7400 \mathrm{~km} \mathrm{~s}^{-1}$ after correcting for the host galaxy's recession velocity. The spectrum does not show any other strong emission features characteristic of normal Type II supernovae at late times (e.g., [O I] and [Ca II]), except for a tentative low signal-to-noise $(\mathrm{S} / \mathrm{N})$ feature centered at $6180 \AA$ with FWHM $\sim 3600 \mathrm{~km} \mathrm{~s}^{-1}$. If the spectrum is heavily smoothed, there is a "bump" that shows up more clearly at $\gtrsim 7000 \AA$, which could correspond to low-level emission associated with the [Ca II] doublet at $\lambda \lambda$ 7291, 7323. There is an increase in flux to the blue of $\sim 5500 \AA$, which could in part be explained by contamination from nearby sources, although the $\mathrm{S} / \mathrm{N}$ in the blue part of the spectrum decreases also due to flat-fielding errors.

The broad and boxy $\mathrm{H} \alpha$ emission feature detected in SN 2008jb is not typically observed in late-time spectra of Type II supernovae. We used spectra of normal Type IIPs in the SUSPECT database to measure the FWHM at $\gtrsim 300$ days after explosion, in the nebular phase, and measure $\mathrm{H} \alpha$ line widths of $\sim 2300-2900 \mathrm{~km} \mathrm{~s}^{-1}$, significantly lower velocities than SN 2008jb. The peculiar SN 2007od (Andrews et al. 2010; Inserra et al. 2011) is the only example we could find in the literature of a Type IIP with a comparably broad and blueshifted $\mathrm{H} \alpha$ component at late times. In this case, however, the line also showed multiple narrower peaks on top of the broad profile that indicated clear signs of interaction between the ejecta and the circumstellar medium (CSM) from the progenitor wind, which are not seen in SN 2008jb.

Other less common Type II supernovae have shown very broad emission lines at late times. The Type IIL SN 1979C in M100 (e.g., Branch et al. 1981) showed a broad $\sim 15,000 \mathrm{~km} \mathrm{~s}^{-1}$ $\mathrm{H} \alpha$ profile one year after discovery (Cappellaro et al. 1995). Also the Type IIL SN 1980K showed an $\mathrm{H} \alpha$ profile with FWHM $10,000 \mathrm{~km} \mathrm{~s}^{-1}$ eight years after the explosion (Fesen \& Becker 1990), although at earlier epochs comparable to our observations of SN 2008jb the line widths were significantly narrower (e.g., Cappellaro et al. 1995). The well-studied Type IIb SN 1993J showed a strong $\mathrm{H} \alpha$ emission feature at $>1$ year after explosion, with a velocity and profile shape fairly similar to SN 2008jb (Filippenko et al. 1994; Matheson et al. 2000). The boxy feature observed in SN 1993J was interpreted as an indication of circumstellar interaction in a spherical shell (Matheson et al. 


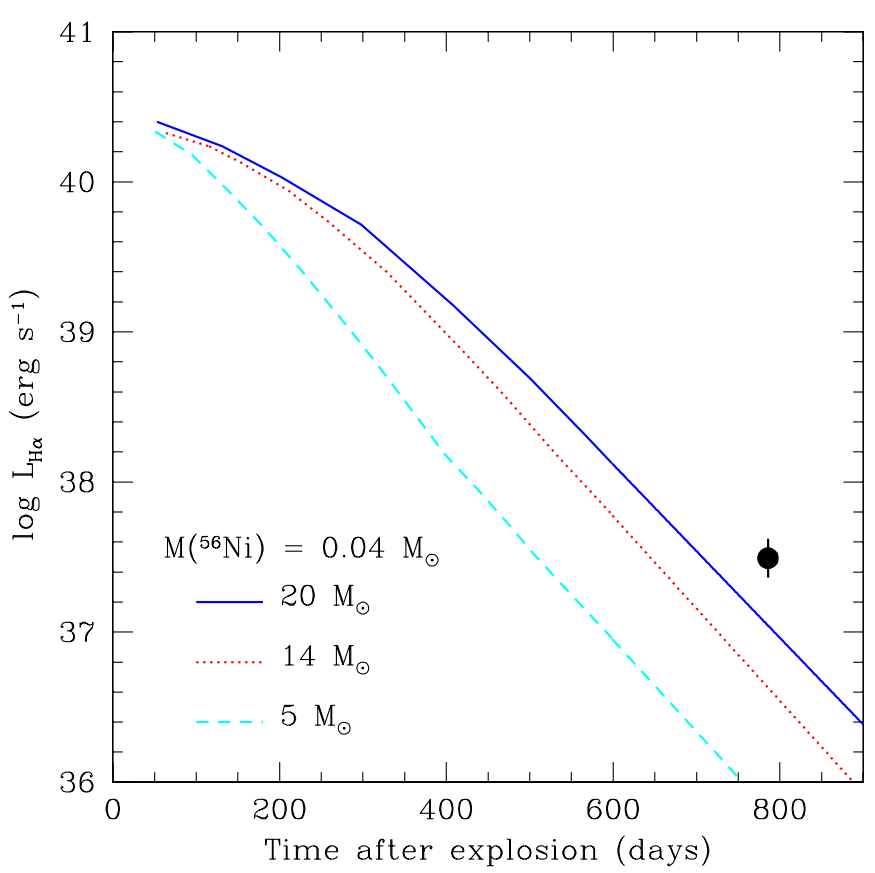

Figure 7. Evolution of the $\mathrm{H} \alpha$ emission line luminosity as a function of time from the models of Chugai (1991) and taken from the parameterization presented in Pastorello et al.(2009b). The filled circle is the measurement from the spectrum of SN 2008jb assuming a conservative error of $30 \%$ (which includes calibration) in luminosity. These models trace the evolution of the $\mathrm{H} \alpha$ line luminosity under the assumption of pure radioactive decay of ${ }^{56} \mathrm{Co}$. The different lines correspond to ejected masses of $20 M_{\odot}$ (continuous line), $14 M_{\odot}$ (dotted line), and $5 M_{\odot}$ (dashed line). Here we have scaled the model curves to a ${ }^{56} \mathrm{Ni}$ mass of $0.04 M_{\odot}$ consistent with SN 2008jb.

(A color version of this figure is available in the online journal.)

2000), which supported the evidence from the radio and X-ray observations (e.g., Fransson et al. 1996, and references therein). Patat et al. (1995) had also shown that the luminosity of the $\mathrm{H} \alpha$ line $0.5-1$ year after the explosion was in excess of the expectations from radioactive ${ }^{56} \mathrm{Co}$ decay, and the most likely source of extra energy was the ejecta-CSM interaction.

Chugai (1991) presented models of the evolution of the $\mathrm{H} \alpha$ line luminosity as a function of time for Type II supernovae assuming radioactive decay as the energy source. We used these models to test if the measured luminosity of the $\mathrm{H} \alpha$ emission line in $\mathrm{SN} 2008 \mathrm{jb}$ at $\sim 800$ days after explosion is consistent with heating from radioactive decay or extra energy is needed to explain it. We use the parameterization of the models presented in Pastorello et al. (2009b; their Figure 9) scaled to a nickel mass of $0.04 M_{\odot}$, and extrapolated the curves linearly at late times (in $\log L$ versus time). Figure 7 shows the models with different assumptions for the total ejected mass from the progenitor $\left(M=5,14,20 M_{\odot}\right)$ and the measured $\mathrm{H} \alpha$ luminosity of SN 2008jb after correcting for Galactic and internal extinction. There is a clear excess in $\mathrm{H} \alpha$ emission luminosity with respect to the models at $\sim 800$ days by a factor of $\sim 1.5$, suggesting the existence of an extra energy source (e.g., from ejecta interaction with the progenitor wind) or a more massive progenitor star $\left(\gtrsim 20 M_{\odot}\right)$. Unfortunately, we do not have early spectra to trace the evolution in $\mathrm{H} \alpha$ emission luminosity with time to differentiate between these scenarios. Also, the gap between CCD chips is right at the wavelength of the $\mathrm{H} \alpha$ line, so we are unable to detect a narrow component that would be a clear indication of circumstellar interaction.

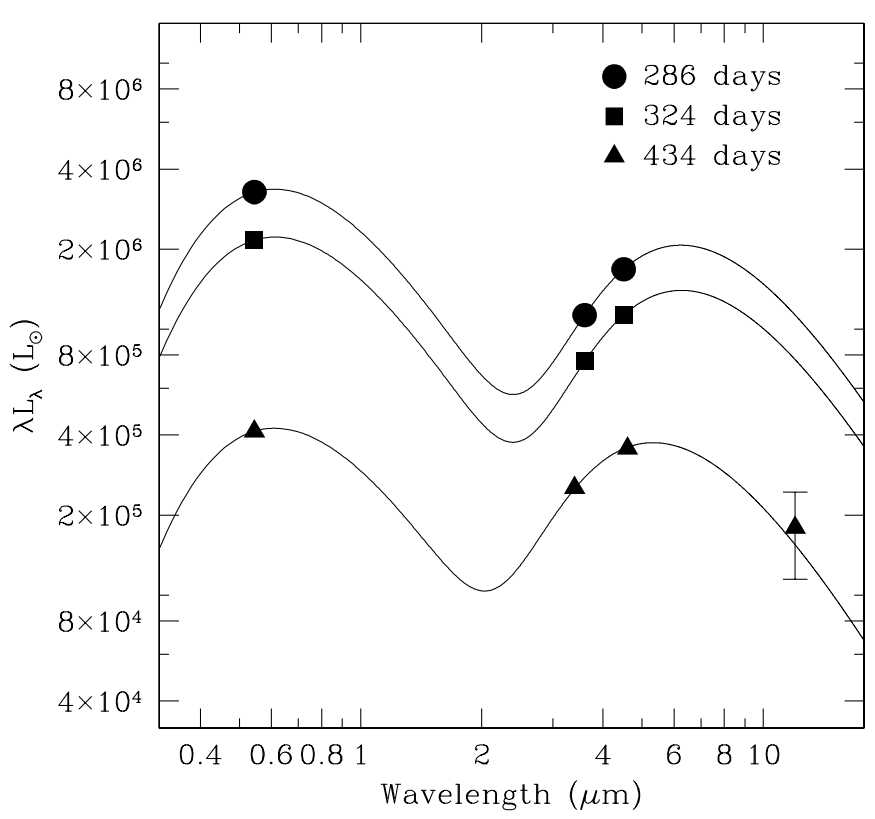

Figure 8. Spectral energy distribution of SN 2008jb at three late-time epochs including $V$-band, Spitzer/IRAC $3.6 \mu \mathrm{m}$ and $4.5 \mu \mathrm{m}$, and WISE $3.4 \mu \mathrm{m}, 4.6 \mu \mathrm{m}$, and $12 \mu \mathrm{m}$ fluxes. The fluxes have been corrected for Galactic and host extinction. The lines are fits to the data using the sum of two blackbodies. For the "hot" blackbody component we assume $T_{\text {hot }}=6000 \mathrm{~K}$ in all three epochs and fit for the absolute normalization. The "warm" component is also a blackbody, but we fit for the absolute normalization and temperature. The temperature of the warm component is between $\sim 600-700 \mathrm{~K}$ in the three epochs. The labels show the epochs (in days) after the first detection.

Table 7

Results from Blackbody Fits to Optical and Mid-IR Data

\begin{tabular}{lcccccc}
\hline \hline $\begin{array}{l}\text { Epoch }^{\mathrm{a}} \\
(\text { days })\end{array}$ & $\begin{array}{c}L_{\text {hot }} \\
\left(10^{6} L_{\odot}\right)\end{array}$ & $\begin{array}{c}R_{\text {hot }} \\
(\mathrm{AU})\end{array}$ & $\begin{array}{c}T_{\text {hot }} \\
(\mathrm{K})\end{array}$ & $\begin{array}{c}L_{\text {warm }} \\
\left(10^{6} L_{\odot}\right)\end{array}$ & $\begin{array}{c}R_{\text {warm }} \\
(\mathrm{AU})\end{array}$ & $\begin{array}{c}T_{\text {warm }} \\
(\mathrm{K})\end{array}$ \\
\hline 286.4 & 4.57 & 9.2 & 6000 & 2.77 & 765 & 581 \\
324.0 & 3.01 & 7.5 & 6000 & 1.07 & 633 & 579 \\
433.9 & 0.58 & 3.3 & 6000 & 0.50 & 237 & 680 \\
\hline
\end{tabular}

Notes. $T_{\text {hot }}$ is fixed in all epochs at $6000 \mathrm{~K}$.

${ }^{\text {a }}$ HJD - 2,454,789.7

\subsection{Mid-IR Emission}

We clearly detect mid-IR emission from SN 2008jb in three Spitzer and WISE epochs obtained between 286 and 434 days after the first optical detection (see Figure 5). The red [3.6]-[4.5] Spitzer color of $1.2 \mathrm{mag}$ indicates a rising spectral energy distribution (SED) at mid-IR wavelengths, a clear sign of warm dust emission. In order to characterize the evolution of the optical and mid-IR emission, we fit the SED of the supernova using two blackbodies, a hot component to fit the optical $V$-band data and a warm component to fit the mid-IR data. Since we only have single-band optical data at late times, we assume an effective temperature of $T_{\text {eff }}=6000 \mathrm{~K}$ for the hot component, which is a typical temperature measured in normal Type II supernovae in the nebular phase.

The results of the two-component blackbody fits are presented in Table 7, and Figure 8 shows the model fits to the SED as a function of time. The total integrated luminosity decreases by a factor of 6.8 in 148 days, which is equivalent to 0.014 mag day $^{-1}$. The luminosity contributed by the warm blackbody component, which is better constrained from the observed SED, decreases by a factor of 5.5 in 148 days, or $0.013 \mathrm{mag} \mathrm{day}^{-1}$. The 
consistency with the late-time $V$-band decline and the ${ }^{56} \mathrm{Co}$ decay slope is interesting and argues for radioactive decay as the dominant energy source of dust heating. The temperature of the warm blackbody component is $600-700 \mathrm{~K}$ in the three epochs where we have mid-IR imaging. The WISE data from day 434 clearly shows that the SED peaks between 4.6 and $12 \mu \mathrm{m}$, supporting our conclusion. We also find that the radius of the warm blackbody component decreases by a factor of $\sim 3$ between the first and last epochs with mid-IR data, between 765 AU (day 286) and 237 AU (day 434). The total mid-IR luminosity can be used to estimate the amount of dust needed to explain it (e.g., Dwek et al. 1983; Prieto et al. 2009); we find a dust mass of $\sim 10^{-5}-10^{-4} M_{\odot}$ assuming a blackbody spectrum and carbon dust composition.

Several nearby Type II supernovae have shown excess mid-IR emission at late times, which is interpreted as the presence of warm dust either newly formed or pre-existing in the progenitor CSM (or a combination). Some examples of nearby Type IIP events with clear signs of warm dust emission include SN 2003gd (Sugerman et al. 2006; Meikle et al. 2007), SN 2004et (Kotak et al. 2009), SN 2004dj (Szalai et al. 2011; Meikle et al. 2011), SN 2007it (Andrews et al. 2011), and SN 2007od (Andrews et al. 2010). The classic, luminous Type IIL SN 1979C and SN 1980K showed infrared excesses at late times (Dwek 1983; Dwek et al. 1983). Also, a large fraction of Type IIn supernovae show mid-IR emission at late times, which has been associated with pre-existing CSM dust (e.g., Fox et al. 2011, and references therein). In the low-luminosity end, SN 2008S-like events have dusty massive star progenitors and also show signs of dust during the transients (e.g., Prieto et al. 2008b, 2009; Thompson et al. 2009; Kochanek 2011).

The typical dust masses needed to explain the mid-IR emission in normal Type II supernovae are $\lesssim 10^{-3} M_{\odot}$ (but see Matsuura et al. 2011 for the discovery of a large reservoir of cold dust in SN 1987A) and fairly consistent with the range derived here for SN 2008jb. The observations for several of the well-studied Type IIs have been usually explained by newly formed dust in the ejecta. It seems unlikely that this can explain $\mathrm{SN} 2008 \mathrm{jb}$. The main reason for this is that the shock velocities inferred from the blackbody fits to the mid-IR detections are in the range of 1000-4500 $\mathrm{km} \mathrm{s}^{-1}$ (depending on the epoch), which is quite low compared to the observed velocity of the $\mathrm{H} \alpha$ emission line. On the other hand, a decreasing blackbody radius as a function of time has been seen in SN 2008S and NGC 300-OT (also the Type IIP SN 2007it and SN 2007od) and has been explained by Kochanek (2011) using a model in which dust reforms in the progenitor wind. This model, however, requires very high densities and low velocities, which are not observed in SN 2008jb.

\subsection{Host Galaxy Environment}

The host of SN 2008jb, ESO 302-14, is a star-forming irregular galaxy similar to the Magellanic Clouds. In particular, it has a total $B$-band absolute magnitude $M_{B}=-15.3 \mathrm{mag}$, $\sim 1$ mag fainter than the SMC, with a low UV derived (total) current SFR of $0.03 M_{\odot} \mathrm{yr}^{-1}$ (Lee et al. 2009). The total stellar mass of the galaxy is $4 \times 10^{7} M_{\odot}$, estimated from $M_{B}, M_{R}$, and the mass-to-light ratios as a function of $B-R$ presented in Bell \& de Jong (2001), assuming a Salpeter IMF. The total neutral hydrogen $(\mathrm{HI})$ mass is $3 \times 10^{8} M_{\odot}$ (Meurer et al. 2006, scaled by our adopted distance). These masses imply high SFRs per unit stellar mass, $\log \left(\mathrm{SFR} / M_{\star}\right) \simeq-9.1$ and $\log \left(\mathrm{SFR} / M_{\mathrm{HI}}\right) \simeq-9.9$, which are typical for local star-forming dwarf galaxies (e.g., Lee et al. 2011).

We use the spectrum we obtained of the HII region at $\sim 150$ pc from the position of SN 2008jb to measure the local oxygen abundance using the strong forbidden oxygen lines and hydrogen recombination lines. Using the emission line fluxes reported in Table 3 and correcting for host galaxy extinction, we estimated the $[\mathrm{O}$ III $] \lambda 5007 / \mathrm{H} \beta$ and $[\mathrm{N}$ II $] / \mathrm{H} \alpha$ line ratios. Then, we used the $N 2$ and $O 3 N 2$ calibrations from Pettini \& Pagel (2004; PP04) to estimate oxygen abundances of $12+\log (\mathrm{O} / \mathrm{H})=$ $8.13 \pm 0.03$ (N2) and $8.21 \pm 0.03$ (O3N2). These uncertainties are statistical and do not include the $0.1-0.2$ dex error in the oxygen abundance calibration (PP04). For comparison, the average oxygen abundance of $\mathrm{HI}$ regions in the SMC from Russell \& Dopita (1990) are $12+\log (\mathrm{O} / \mathrm{H})=8.08$ (N2) and $8.11($ O3N2).

The environment of SN 2008jb in ESO 302-14 has a low oxygen abundance compared to the environments of nearby Type IIP supernovae $(\lesssim 30 \mathrm{Mpc})$ used to constrain progenitor properties from deep the pre-explosion observations (Smartt et al. 2009), although there are also normal core-collapse supernovae discovered in relatively nearby galaxies that have fairly low-metallicity environments (e.g., Prieto et al. 2008b; Anderson et al. 2010). The metallicity here is similar to the environments of long-duration gamma-ray burst (GRB) hosts (e.g., Stanek et al. 2006; Levesque et al. 2010) and the hosts of the most luminous core-collapse supernovae that are being discovered in galaxy-blind surveys (e.g., Kozłowski et al. 2010; Stoll et al. 2011), which are bound to contain a population of very massive stars.

By closely examining the local host galaxy properties we can constrain the progenitor properties (see, e.g., Anderson \& James 2008, 2009). We have relatively deep optical images obtained at late times with Magellan/IMACS, archival UV data from $G A L E X$, and $\mathrm{H} \alpha$ from SINGG. Figure 9 shows a mosaic with the Magellan $R$-band and GALEX FUV image, scaled to fit the whole galaxy (top panels) and the region where the supernova exploded (lower panels). We see that SN 2008jb exploded in a large star formation complex, the brightest and highest surface brightness star-forming region within the galaxy in the optical and FUV. It is composed of at least two resolved clusters or stellar associations that are well separated in the Magellan $R$-band image. Interestingly, the $\mathrm{H} \alpha$ emission (red contours in the lower panel of Figure 9) is offset and outside the brightest optical and FUV emission, forming an apparent ring with a projected diameter of $\simeq 700 \mathrm{pc}$.

We can estimate an approximate age for the star-forming complex in ESO 302-14 using the ratio of $\mathrm{H} \alpha$ to FUV luminosities, which is a sensitive age indicator in an instantaneous burst of star formation (e.g., Stewart et al. 2000; Sánchez-Gil et al. 2011). We use the Starburst99 (Leitherer et al. 1999) models to generate a grid of single-age clusters with total masses between $5 \times 10^{4} M_{\odot}$ and $4 \times 10^{6} M_{\odot}$, in steps of $10^{5} M_{\odot}$. We choose a Salpeter IMF with stellar masses between 0.1 and $100 M_{\odot}$ and Geneva stellar evolution models with $Z=0.004$, consistent with the measured oxygen abundance of the $\mathrm{H}$ II region. Figure 10 shows the expected ratio of $\mathrm{H} \alpha$-to-FUV luminosities as a function of the FUV luminosities for these models. We label the ages of the clusters between $1 \mathrm{Myr}$ and $50 \mathrm{Myr}$. The total measured $\mathrm{H} \alpha$-toFUV ratio $\log \left(L_{\mathrm{H} \alpha} / L_{\mathrm{FUV}}\right)=12.41 \pm 0.16$ and FUV luminosity $L_{\mathrm{FUV}}=(5.7 \pm 1.8) \times 10^{25} \mathrm{erg} \mathrm{s}^{-1} \mathrm{~Hz}^{-1}$ of the star-forming complex, corrected by Galactic and host galaxy extinction, are shown in the figure. We obtain an age of $8.8 \pm 0.8 \mathrm{Myr}$ and 


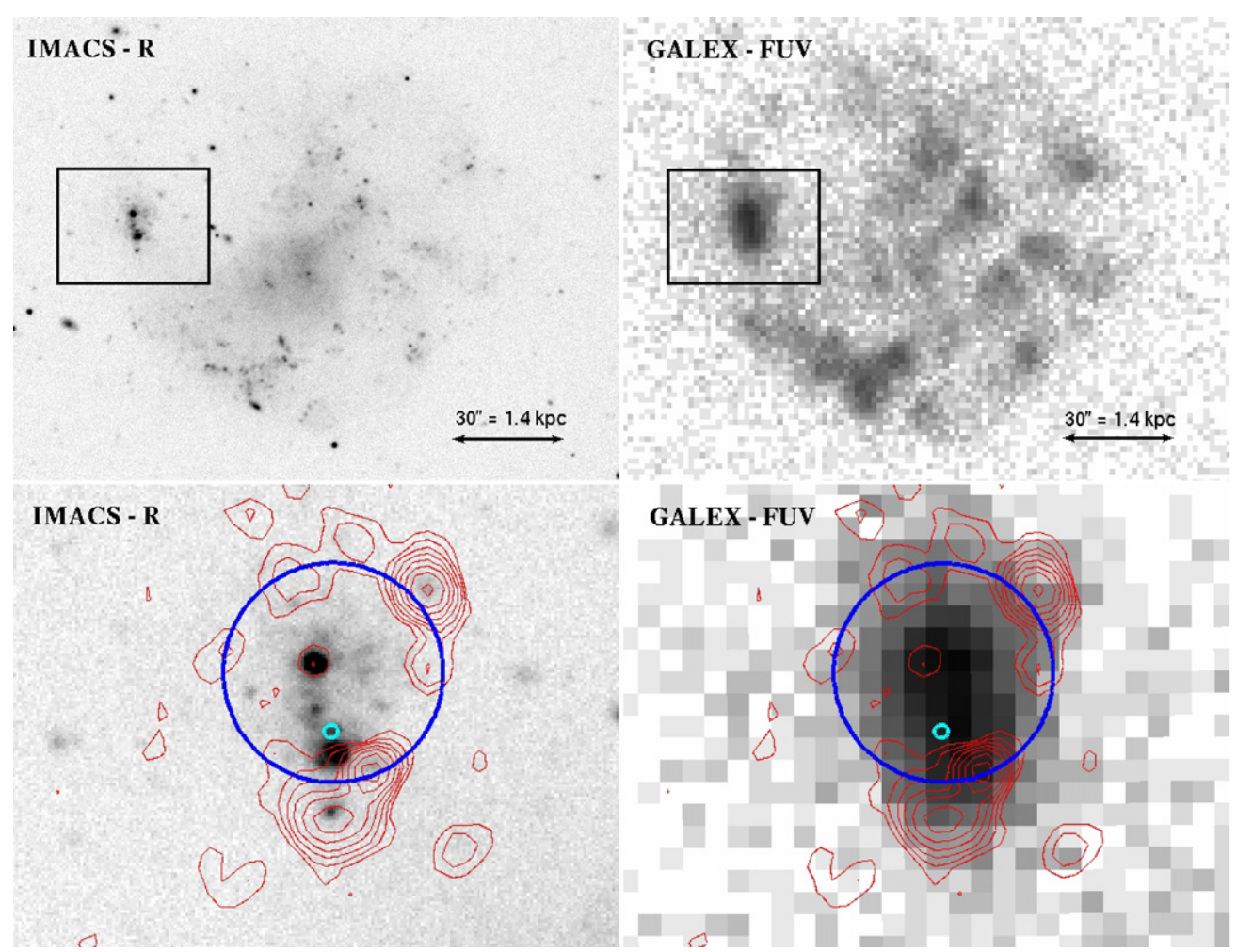

Figure 9. Host galaxy environment of SN 2008jb. Top panels: Magellan/IMACS $R$-band image obtained on 2010 November 10 (left) and GALEX/FUV $\left(\lambda_{\text {eff }}=1539 \AA\right)$ archival image obtained on 2004 November 18 (right). The FWHM of stars in the images are 0'.7 ( $R$-band) and 4" (FUV). Bottom panels: zoomed-in versions of the rectangular regions around the supernova position shown in the top panels. The red contours trace H $\alpha$ emission from archival images obtained by the SINGG survey on 2000 October 28 . The blue circle has a diameter of $15^{\prime \prime} \approx 700 \mathrm{pc}$ and approximately follows the H $\alpha$ emission around the star formation complex. The cyan circle shows the position of SN 2008jb. In all panels, north is up and east is to the left. (A color version of this figure is available in the online journal.)

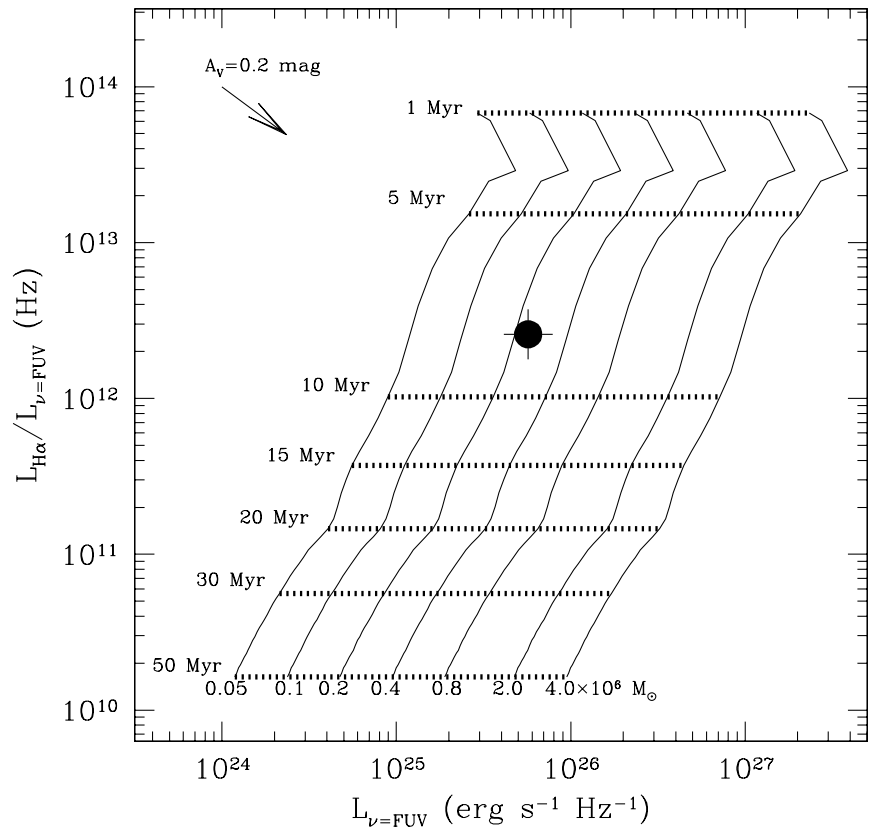

Figure 10. H $\alpha$ /FUV ratio as a function of the specific FUV luminosity. The filled circle shows the integrated value for the star formation complex that hosts SN 2008jb in ESO 302-14, after correcting for the total extinction. The lines show theoretical Starburst99 models for single-burst clusters with masses $5 \times 10^{4}-4 \times 10^{6} M_{\odot}$ and ages in the range $1-50 \mathrm{Myr}$, assuming a metallicity of $0.2 Z_{\odot}$ and a Salpeter IMF. If the complex that hosts SN 2008jb is from a single burst of star formation, it is consistent with having a total mass $M=(2.4 \pm 1.0) \times 10^{5} M_{\odot}$ and age $8.8 \pm 0.8 \mathrm{Myr}$. The vector in the top left shows the effect of $A_{V}=0.2 \mathrm{mag}$ of host extinction in the fluxes. total mass of $(2.4 \pm 1.0) \times 10^{5} M_{\odot}$. We also used the integrated $R$-band flux (extinction corrected) of the star-forming region to independently check this mass estimate. We derive a total mass of $1.9 \times 10^{5} M_{\odot}$ from the $R$-band flux and the mass-to-light ratio obtained from the Starburst99 models, which is fully consistent with the FUV estimate.

If the progenitor star of SN 2008jb was formed in this star formation episode $9 \mathrm{Myr}$ ago, then we infer an initial main-sequence mass of $\simeq 23 M_{\odot}$ for the progenitor from the Geneva stellar evolution models with extended mass loss used in Starburst99 (Lejeune \& Schaerer 2001). This estimate is obtained as the maximum main-sequence mass of a star from an isochrone of $9 \mathrm{Myr}$ of age. We note that this estimate is based only in the total $\mathrm{H} \alpha$ and FUV fluxes of the region, and a more detailed analysis of the SEDs of the individual stellar clusters is in preparation.

The equivalent width $(\mathrm{EW})$ of the $\mathrm{H} \alpha(\mathrm{EW}=263 \AA)$ and $\mathrm{H} \beta(=53 \AA)$ emission lines in the spectrum of the $\mathrm{H}_{\text {II }}$ region can also be used to estimate an age for the star-forming region using the Starburst99 models (e.g., Stasińska \& Leitherer 1996; Schaerer \& Vacca 1998; Leloudas et al. 2011). Essentially, the EW of the recombination lines gives an estimate of the young over the old stellar population (see Leitherer 2005, and references therein). We obtain an age of $6 \mathrm{Myr}$ from $\mathrm{H} \alpha \mathrm{EW}$ and $5 \mathrm{Myr}$ from $\mathrm{H} \beta \mathrm{EW}$. These estimates are 3-4 Myr $(\sim 3 \sigma$ using the statistical uncertainty) younger than the age derived from the $\mathrm{H} \alpha$-to-FUV ratio of the whole star-forming region. The differences might imply multiple star formation episodes within the star-forming region, however, this would need to be 
tested with a detailed study of the stellar populations within the region.

We can estimate the SFR of the star-forming region using the FUV luminosity with the calibrations of Lee et al. (2009), finding $\mathrm{SFR}_{\mathrm{UV}}=0.01 M_{\odot} \mathrm{yr}^{-1}$, or about $30 \%$ of the total SFR of the dwarf galaxy. We note that the $\mathrm{H} \alpha$-estimated SFR is a factor of $\sim 8$ lower than the FUV value, a systematic difference seen in dwarf galaxies at low SFRs which lacks a satisfactory explanation (e.g., Lee et al. 2009; see also Fumagalli et al. 2011).

\section{SUMMARY AND CONCLUSIONS}

We have presented the discovery, follow-up observations, and analysis of SN 2008jb, a bright Type II supernova in the metalpoor, southern dwarf irregular galaxy ESO 302-14 at $\sim 10 \mathrm{Mpc}$. This 13.6 mag supernova was found in archival data obtained by the CRTS and ASAS all-sky surveys. This transient was missed by galaxy-targeted supernova surveys like CHASE and by amateur astronomers mainly because the host galaxy is a lowluminosity dwarf, $\sim 1$ mag fainter than the SMC, and targeted surveys use catalogs that are incomplete for small galaxies (e.g., Leaman et al. 2011).

SN 2008 jb has $V$ - and $I$-band light curves similar to normal Type IIP supernovae, with peak magnitude $M_{V} \simeq-16.5 \mathrm{mag}$, but it can also be classified as an intermediate case between Type IIP and Type IIL due to its faster initial decay, perhaps similar to SN 1999ga (Pastorello et al. 2009b). It shows a $\sim 95$ day plateau and a fast $\sim 1.4$ mag decline to a late-time decline slope of $0.013 \mathrm{mag}^{-1} \mathrm{day}^{-1}$ in the $V$ band. This decline is consistent with the radioactive decay of ${ }^{56} \mathrm{Co}$ to ${ }^{56} \mathrm{Fe}$, and argues for $0.04 \pm 0.01 M_{\odot}$ of ${ }^{56} \mathrm{Ni}$ synthesized in the explosion that is powering the light curve. We detect mid-IR emission from SN 2008jb 8-14 months after explosion in three epochs of archival Spitzer and WISE data. The mid-IR emission has an SED with blackbody temperature of 600-700 K, characteristic of the warm dust emission seen in some nearby core-collapse supernovae and luminous transients (e.g., Kotak et al. 2009; Prieto et al. 2009). The evolution of the mid-IR emission with time is consistent with the products of radioactive decay heating the dust. The characteristic mid-IR dust radius shrinks with time, an evolution that is not typically seen in normal Type IIs (some exceptions are SN 2007it and SN 2007od; Andrews et al. 2010, 2011).

We obtained a spectrum of SN 2008jb about two years after the explosion. It displays a very broad (FWHM $\simeq$ $14,000 \mathrm{~km} \mathrm{~s}^{-1}$ ), boxy, and flat-topped $\mathrm{H} \alpha$ emission line, leading to its Type II supernova spectroscopic classification. The broad and boxy line profile seen in $\mathrm{H} \alpha$ is quite unusual for normal Type IIP supernovae at late times, but has been seen in some objects like SN 1993J, SN 2007od, and also a few well-studied Type IIL supernovae. We find that the $\mathrm{H} \alpha$ line luminosity is in excess of the expected luminosity from radioactive ${ }^{56} \mathrm{Co}$ decay predicted by the models of Chugai (1991) for total ejected masses $M=5,14,20 M_{\odot}$. This indicates that there is an external source of energy, like ejecta-CSM interaction, and/or the mass of the progenitor star is $\gtrsim 20 M_{\odot}$. We do not see clear signs of ejecta-CSM interaction like narrow lines or irregularities in the $\mathrm{H} \alpha$ profile. It would be interesting to obtain late time $\mathrm{X}$-ray and radio observations in order to have independent constraints on the importance of ejecta-CSM interaction in SN 2008jb.

We studied the host galaxy environment of SN 2008jb in ESO 302-14 with optical spectra and imaging. Using the spec- trum of an $\mathrm{H}$ II region at $\sim 150$ pc from the supernova site we measure an oxygen abundance of $12+\log (\mathrm{O} / \mathrm{H})=8.21 \pm 0.03$ (PP04 O3N2 method) and $12+\log (\mathrm{O} / \mathrm{H})=8.13 \pm 0.03$ (PP04 N2 method) from the strong nebular emission lines, which is similar to the SMC and one of the lowest measured metallicities of local core-collapse supernovae environments (e.g., in the lower $3 \%$ of measured oxygen abundances of the core-collapse sample of Anderson et al. 2010). The supernova exploded in a large star-forming complex with strong optical and GALEX FUV emission which is surrounded by a large $\mathrm{H} \alpha$ ring with $R \simeq 350 \mathrm{pc}$. The $\mathrm{H} \alpha$-to-FUV ratio of this region is consistent with a stellar population with an age of $\sim 9 \mathrm{Myr}$ derived from Starburst99 modeling and single-age stellar population models. This age implies a supernova progenitor mass of $\simeq 23 M_{\odot}$, assuming a single star (but see, e.g., Smith et al. 2011 a for the importance of binary progenitors), if the progenitor formed in this star-forming episode. The $\mathrm{EW}$ of the $\mathrm{H} \alpha$ and $\mathrm{H} \beta$ emission lines in the spectrum of the $\mathrm{H}$ II region gives another constraint on the age of the region of 5-6 Myr, 3-4 Myr younger than the estimate from $\mathrm{H} \alpha$-to-FUV ratio. We note that the star formation history could be more complicated than a single burst, and we plan to study the region in detail using multiwavelength data in a future study. In particular, it would be interesting to include high-resolution data from $H S T$.

Large expanding $\mathrm{H} \alpha$ shells (supershells with radii $>300 \mathrm{pc}$ ) have been observed and studied in many nearby star-forming dwarf galaxies and have typical dynamical ages of $\sim 10 \mathrm{Myr}$ (e.g., Martin 1998), which is fairly consistent with the age we derive here from the $\mathrm{H} \alpha$ and FUV emission. These structures are produced by the combined effect of many supernova explosions and winds from massive stars (e.g., Chakraborti \& Ray 2011), and are the likely precursors of galactic winds. In a sense, we may perhaps be witnessing supernova feedback in real time in this star-forming region.

The bias to large star-forming galaxies is clearly present in the samples of nearby ( $\lesssim 30 \mathrm{Mpc}$ ) core-collapse supernovae used for progenitor studies (e.g., Smartt et al. 2009) and has been discussed in detail as a possible explanation for the discrepancy between measured local supernova rates and predicted supernova rates from galaxy SFRs (e.g., Horiuchi et al. 2011). Since the environments of nearby core-collapse supernovae used for progenitor studies generally miss dwarf galaxies because they were not included in the original searches, the progenitor properties and conclusions drawn from these samples regarding stellar evolution are not complete. In particular, the dearth of high-mass ( 20-30 $M_{\odot}$ ) progenitor stars of Type IIP supernovae ("red supergiant problem") could be alleviated if these progenitor stars prefer lower-metallicity environments. For example, this could be caused by environmental variations in the stellar IMF (e.g., Meurer et al. 2009) or by changes in the fraction of Type II spectroscopic subtypes as a function of metallicity due to stellar evolution (e.g., Arcavi et al. 2010). Indeed, we find that the properties of the spectrum of SN 2008jb are more consistent with a massive progenitor (but see discussion about supernova modeling in, e.g., Smartt et al. 2009; Bersten \& Hamuy 2009; Bersten et al. 2011), and the star-forming region where it was found has a young age compared with the ages of detected Type IIP progenitors ( $\gtrsim 15 \mathrm{Myr}$ ).

Interestingly, a strong preference for low-metallicity hosts is observed in long GRBs (e.g., Stanek et al. 2006) and luminous core-collapse supernovae (e.g., Neill et al. 2011; Stoll et al. 2011), which have been linked with massive star progenitors $\left(M \gtrsim 20-30 M_{\odot}\right)$. SN 2008jb offers the unique 
chance of studying in detail a nearby Type II supernova with host properties similar to long GRBs and the most luminous core-collapse supernovae.

The mapping between different classes of massive stars and their supernovae is not yet fully understood. Special insights are expected to be obtained when unusual explosions can be connected to unusual progenitor stars and galaxy hosts. Nearby objects are especially useful in terms of larger fluxes for an extended time after explosion, better spatial resolution for progenitor studies, and improved prospects for detection by new messengers like gamma rays (e.g., Timmes \& Woosley 1997; Horiuchi \& Beacom 2010), neutrinos (e.g., Ando \& Beacom 2005; Kistler et al. 2011), and gravitational waves (e.g., Ott 2009). In addition, data from these objects are needed for a comprehensive understanding of the nearby universe.

It is difficult to find the nearest supernovae in small host galaxies with searches that target individual (generally large) galaxies, like LOSS, CHASE, and also amateur efforts. It is also difficult to find them with volume-based searches such as the Palomar Transient Factory (Rau et al. 2009) and PanSTARRS (Kaiser et al. 2002) which have a deep, but relatively small survey area with good cadence. A shallower all-sky survey with excellent cadence, like ASAS, will help us find nearby ( $\lesssim 30 \mathrm{Mpc}$ ) supernovae in all kinds of environments, including low-metallicity dwarf galaxies like the host of SN 2008jb (see also Khan et al. 2011; Stoll et al. 2011, for other supernovae studied with ASAS). Upgrades that will significantly increase the sensitivity and response speed of ASAS are underway.

We thank John Mulchaey for obtaining one of the Magellan images presented in this work; Chris Kochanek for detailed comments; Rupali Chandar, Crystal Martin, and Linda Watson for discussions; and Chris Burns for providing his image subtraction code. We also thank the anonymous referee for a careful reading of the manuscript. We are indebted to the staff of Las Campanas Observatory for their assistance. J.L.P. acknowledges support from NASA through Hubble Fellowship Grant HF-51261.01-A awarded by STScI, which is operated by AURA, Inc. for NASA, under contract NAS 5-2655. J.F.B. is supported by the National Science Foundation CAREER Grant PHY-0547102. G.P. is supported by the Polish MNiSW Grant N203 007 31/1328. K.S.Z. and D.M.S. are supported in part by NSF Grant AST-0908816. Support for M.C. is provided by the Ministry for the Economy, Development, and Tourism's Programa Iniciativa Científica Milenio through grant P07-021-F, awarded to The Milky Way Millenium Nucleus; by Proyecto Basal PFB-06/2007; by FONDAP Centro de Astrofísica 15010003; and by proyecto FONDECYT Regular \#1110326. The CRTS is supported in part by the NSF Grant AST-0909182. This research has made extensive use of the NASA/IPAC Extragalactic Database (NED) which is operated by the JPL, Caltech, under contract with NASA.

\section{REFERENCES}

Aldering, G., Humphreys, R. M., \& Richmond, M. 1994, AJ, 107, 662 Anderson, J. P., Covarrubias, R. A., James, P. A., Hamuy, M., \& Habergham, S. M. 2010, MNRAS, 407, 2660

Anderson, J. P., \& James, P. A. 2008, MNRAS, 390, 1527

Anderson, J. P., \& James, P. A. 2009, MNRAS, 399, 559

Ando, S., \& Beacom, J. F. 2005, Phys. Rev. Lett., 95, 061103

Andrews, J. E., Gallagher, J. S., Clayton, G. C., et al. 2010, ApJ, 715, 541

Andrews, J. E., Sugerman, B. E. K., Clayton, G. C., et al. 2011, ApJ, 731, 47

Arcavi, I., Gal-Yam, A., Kasliwal, M. M., et al. 2010, ApJ, 721, 777
Arnett, W. D., Bahcall, J. N., Kirshner, R. P., \& Woosley, S. E. 1989, ARA\&A, 27, 629

Barbon, R., Ciatti, F., \& Rosino, L. 1979, A\&A, 72, 287

Bell, E. F., \& de Jong, R. S. 2001, ApJ, 550, 212

Bersten, M. C., Benvenuto, O., \& Hamuy, M. 2011, ApJ, 729, 61

Bersten, M. C., \& Hamuy, M. 2009, ApJ, 701, 200

Branch, D., Falk, S. W., Uomoto, A. K., et al. 1981, ApJ, 244, 780

Calzetti, D. 2001, PASP, 113, 1449

Cappellaro, E., Danziger, I. J., della Valle, M., Gouiffes, C., \& Turatto, M. 1995, A\&A, 293, 723

Cardelli, J. A., Clayton, G. C., \& Mathis, J. S. 1989, ApJ, 345, 245

Chakraborti, S., \& Ray, A. 2011, ApJ, 728, 24

Chugai, N. N. 1991, MNRAS, 250, 513

Crockett, R. M., Eldridge, J. J., Smartt, S. J., et al. 2008, MNRAS, 391, L5

D’Andrea, C. B., Sako, M., Dilday, B., et al. 2010, ApJ, 708, 661

Djorgovski, S. G., et al. 2011, in Proc. The First Year of MAXI: Monitoring Variable X-ray Sources, ed. T. Mihara \& N. Kawai (Tokyo: JAXA Special Publ.), in press (arXiv:1102.5004)

Drake, A. J., Djorgovski, S. G., Mahabal, A., et al. 2009, ApJ, 696, 870

Dressler, A., Hare, T., Bigelow, B. C., \& Osip, D. J. 2006, Proc. SPIE, 6269, 13 Dwek, E. 1983, ApJ, 274, 175

Dwek, E., A'Hearn, M. F., Becklin, E. E., et al. 1983, ApJ, 274, 168

Elias-Rosa, N., Van Dyk, S. D., Li, W., et al. 2009, ApJ, 706, 1174

Elias-Rosa, N., Van Dyk, S. D., Li, W., et al. 2010, ApJ, 714, L254

Fazio, G. G., Hora, J. L., Allen, L. E., et al. 2004, ApJS, 154, 10

Fesen, R. A., \& Becker, R. H. 1990, ApJ, 351, 437

Filippenko, A. V., Li, W. D., Treffers, R. R., \& Modjaz, M. 2001, in ASP Conf. Ser. Vol. 246, IAU Colloq. 183, Small Telescope Astronomy on Global Scales, ed. B. Paczynski, W.-P. Chen, \& C. Lemme (San Francisco, CA: ASP), 121

Filippenko, A. V., Matheson, T., \& Barth, A. J. 1994, AJ, 108, 2220

Fox, O. D., Chevalier, R. A., Skrutskie, M. F., et al. 2011, ApJ, 741, 7

Fransson, C., Lundqvist, P., \& Chevalier, R. A. 1996, ApJ, 461, 993

Fraser, M., Ergon, M., Eldridge, J. J., et al. 2011, MNRAS, 417, 1417

Freedman, W. L., Burns, C. R., Phillips, M. M., et al. 2009, ApJ, 704, 1036

Fumagalli, M., da Silva, R. L., \& Krumholz, M. R. 2011, ApJ, 741, L26

Gal-Yam, A., \& Leonard, D. C. 2009, Nature, 458, 865

Gal-Yam, A., Leonard, D. C., Fox, D. B., et al. 2007, ApJ, 656, 372

Gordon, K. D., Clayton, G. C., Misselt, K. A., Landolt, A. U., \& Wolff, M. J. 2003, ApJ, 594, 279

Hamuy, M. 2003, ApJ, 582, 905

Hamuy, M., Pinto, P. A., Maza, J., et al. 2001, ApJ, 558, 615

Høg, E., Fabricius, C., Makarov, V. V., et al. 2000, A\&A, 355, L27

Horiuchi, S., \& Beacom, J. F. 2010, ApJ, 723, 329

Horiuchi, S., Beacom, J. F., Kochanek, C. S., et al. 2011, ApJ, 738, 154

Inserra, C., Turatto, M., Pastorello, A., et al. 2011, MNRAS, 417, 261

James, P. A., Knapen, J. H., Shane, N. S., Baldry, I. K., \& de Jong, R. S. 2008, A\&A, 482, 507

Kaiser, N., Aussel, H., Burke, B. E., et al. 2002, Proc. SPIE, 4836, 154

Khan, R., Prieto, J. L., Pojmański, G., et al. 2011, ApJ, 726, 106

Kistler, M. D., Yüksel, H., Ando, S., Beacom, J. F., \& Suzuki, Y. 2011, Phys. Rev. D, 83, 123008

Kochanek, C. S. 2011, ApJ, 741, 37

Kochanek, C. S., Beacom, J. F., Kistler, M. D., et al. 2008, ApJ, 684, 1336

Kochanek, C. S., Szczygiel, D. M., \& Stanek, K. Z. 2011, ApJ, 737, 76

Koribalski, B. S., Staveley-Smith, L., Kilborn, V. A., et al. 2004, AJ, 128, 16

Kotak, R., Meikle, W. P. S., Farrah, D., et al. 2009, ApJ, 704, 306

Kozłowski, S., Kochanek, C. S., Stern, D., et al. 2010, ApJ, 722, 1624

Landolt, A. U. 1983, AJ, 88, 439

Lauberts, A., \& Valentijn, E. A. 1989, The Surface Photometry Catalogue of the ESO-Uppsala Galaxies (Garching: European Southern Observatory)

Leaman, J., Li, W., Chornock, R., \& Filippenko, A. V. 2011, MNRAS, 412, 1419

Lee, J. C., Gil de Paz, A., Kennicutt, R. C., Jr., et al. 2011, ApJS, 192, 6

Lee, J. C., Gil de Paz, A., Tremonti, C., et al. 2009, ApJ, 706, 599

Leitherer, C. 2005, in AIP Conf. Vol. 783, The Evolution of Starbursts, ed. E. Manthey, D. Bomans, \& K. Weis (Melville, NY: AIP), 280

Leitherer, C., Schaerer, D., Goldader, J. D., et al. 1999, ApJS, 123, 3

Lejeune, T., \& Schaerer, D. 2001, A\&A, 366, 538

Leloudas, G., Gallazzi, A., Sollerman, J., et al. 2011, A\&A, 530, A95

Levesque, E. M., Kewley, L. J., Berger, E., \& Zahid, H. J. 2010, AJ, 140, 1557

Levesque, E. M., Massey, P., Olsen, K. A. G., et al. 2006, ApJ, 645, 1102

Li, W., Leaman, J., Chornock, R., et al. 2011, MNRAS, 412, 1441

Li, W., Van Dyk, S. D., Filippenko, A. V., \& Cuillandre, J.-C. 2005, PASP, 117 , 121

Maguire, K., di Carlo, E., Smartt, S. J., et al. 2010, MNRAS, 404, 981

Martin, C. L. 1998, ApJ, 506, 222 
Martin, D. C., Fanson, J., Schiminovich, D., et al. 2005, ApJ, 619, L1 Matheson, T., Filippenko, A. V., Ho, L. C., et al. 2000, AJ, 120, 1499

Matsuura, M., Dwek, E., Meixner, M., et al. 2011, Science, 333, 1258

Maund, J. R., Fraser, M., Ergon, M., et al. 2011, ApJ, 739, L37

Maund, J. R., Smartt, S. J., Kudritzki, R. P., Podsiadlowski, P., \& Gilmore, G. F. 2004, Nature, 427, 129

Meikle, W. P. S., Kotak, R., Farrah, D., et al. 2011, ApJ, 732, 109

Meikle, W. P. S., Mattila, S., Pastorello, A., et al. 2007, ApJ, 665, 608

Meurer, G. R., Hanish, D. J., Ferguson, H. C., et al. 2006, ApJS, 165, 307

Meurer, G. R., Wong, O. I., Kim, J. H., et al. 2009, ApJ, 695, 765

Modjaz, M. 2011, Astron. Nachr., 332, 434

Myers, A. T., Krumholz, M. R., Klein, R. I., \& McKee, C. F. 2011, ApJ, 735, 49

Neill, J. D., Sullivan, M., Gal-Yam, A., et al. 2011, ApJ, 727, 15

Nugent, P., Sullivan, M., Ellis, R., et al. 2006, ApJ, 645, 841

Ott, C. D. 2009, Class. Quantum Gravity, 26, 063001

Pastorello, A., Crockett, R. M., Martin, R., et al. 2009a, A\&A, 500, 1013

Pastorello, A., Valenti, S., Zampieri, L., et al. 2009b, MNRAS, 394, 2266

Patat, F., Chugai, N., \& Mazzali, P. A. 1995, A\&A, 299, 715

Paturel, G., Petit, C., Prugniel, Ph., et al. 2003, A\&A, 412, 45

Pettini, M., \& Pagel, B. E. J. 2004, MNRAS, 348, L59

Pignata, G., Maza, J., Antezana, R., et al. 2009, AIP Conf. Ser. 1111, Probing Stellar Populations Out to the Distant Universe: Cefalù 2008, ed. L. A. Antonelli et al. (Melville, NY: AIP), 551

Pojmanski, G. 1998, Acta Astron., 48, 35

Pojmanski, G. 2002, Acta Astron., 52, 397

Poznanski, D., Butler, N., Filippenko, A. V., et al. 2009, ApJ, 694, 1067

Prieto, J. L., Drake, A. J., McNaught, R., \& Garradd, G. 2011, CBET, 2771, 1

Prieto, J. L., Kistler, M. D., Thompson, T. A., et al. 2008a, ApJ, 681, L9

Prieto, J. L., Sellgren, K., Thompson, T. A., \& Kochanek, C. S. 2009, ApJ, 705, 1425

Prieto, J. L., Stanek, K. Z., \& Beacom, J. F. 2008b, ApJ, 673, 999

Rau, A., Kulkarni, S. R., Law, N. M., et al. 2009, PASP, 121, 1334
Russell, S. C., \& Dopita, M. A. 1990, ApJS, 74, 93

Sánchez-Gil, M. C., Jones, D. H., Pérez, E., et al. 2011, MNRAS, 415, 753

Schaerer, D., \& Vacca, W. D. 1998, ApJ, 497, 618

Schlegel, D. J., Finkbeiner, D. P., \& Davis, M. 1998, ApJ, 500, 525

Sheth, K., Regan, M., Hinz, J. L., et al. 2010, PASP, 122, 1397

Smartt, S. J., Eldridge, J. J., Crockett, R. M., \& Maund, J. R. 2009, MNRAS, 395, 1409

Smith, N., Hinkle, K. H., \& Ryde, N. 2009, AJ, 137, 3558

Smith, N., Li, W., Filippenko, A. V., \& Chornock, R. 2011a, MNRAS, 412, 1522

Smith, N., Li, W., Miller, A. A., et al. 2011b, ApJ, 732, 63

Smith, N., Li, W., Silverman, J. M., Ganeshalingam, M., \& Filippenko, A. V. 2011c, MNRAS, 415, 773

Stanek, K. Z., Gnedin, O. Y., Beacom, J. F., et al. 2006, Acta Astron., 56, 333

Stasińska, G., \& Leitherer, C. 1996, ApJS, 107, 661

Stetson, P. B. 1992, in ASP Conf. Ser. 25, Astronomical Data Analysis Software and Systems I, ed. D. M. Worrall, C. Biemesderfer, \& J. Barnes (San Francisco, CA: ASP), 297

Stewart, S. G., Fanelli, M. N., Byrd, G. G., et al. 2000, ApJ, 529, 201

Stoll, R., Prieto, J. L., Stanek, K. Z., et al. 2011, ApJ, 730, 34

Storey, P. J., \& Hummer, D. G. 1995, MNRAS, 272, 41

Sugerman, B. E. K., Ercolano, B., Barlow, M. J., et al. 2006, Science, 313 196

Szalai, T., Vinkó, J., Balog, Z., et al. 2011, A\&A, 527, A61

Szczygiel, D., Khan, R., \& Kochanek, C. S. 2011, ATel, 3431, 1

Thompson, T. A., Prieto, J. L., Stanek, K. Z., et al. 2009, ApJ, 705, 1364

Timmes, F. X., \& Woosley, S. E. 1997, ApJ, 489, 160

Van Dyk, S. D., Li, W., Cenko, S. B., et al. 2011, ApJ, 741, L28

Walmswell, J. J., \& Eldridge, J. J. 2011, MNRAS, in press (arXiv:1109.4637)

Williams, B. F., Dalcanton, J. J., Johnson, L. C., et al. 2011, ApJ, 734, L22

Wright, E. L., Eisenhardt, P. R. M., Mainzer, A. K., et al. 2010, AJ, 140, 1868

Yoon, S.-C., \& Cantiello, M. 2010, ApJ, 717, L62

Young, D. R., Smartt, S. J., Mattila, S., et al. 2008, A\&A, 489, 359 